\title{
An improved simulation model to describe the temperature-dependent population dynamics of the grain aphid, Sitobion avenae
}

\author{
Catriona Duffy ${ }^{\mathrm{a}, *}$, Rowan Fealy ${ }^{\mathrm{b}}$, Reamonn M. Fealy ${ }^{\mathrm{c}}$ \\ a Irish Climate Analysis and Research Units (ICARUS), Department of Geography, Maynooth University, Ireland \\ ${ }^{\mathrm{b}}$ Department of Geography, Maynooth University, Ireland \\ ' Teagasc, Rural Economy and Development Programme, Ashtown, Dublin, Ireland
}

\section{A R T I C L E I N F O}

\section{Article history:}

Received 15 July 2016

Received in revised form 23 January 2017

Accepted 13 March 2017

Available online 10 April 2017

\section{Keywords:}

Aphid

Process-based simulation models

Temperature

Population dynamics

\begin{abstract}
A B S T R A C T
Effective decision support tools are required in order to provide agricultural practitioners with advice regarding appropriate and economic pest management strategies. Process-based simulation models could enhance farmer's abilities to make knowledge-based decisions regarding both the timing and extent to which chemical management is relied upon in an agronomical context. This work describes a new simulation model that quantifies the size and timing of grain aphid populations in response to temperature. The simulation model is comprised of compartmentalised aspects of the aphid's life cycle, which interact with one another to produce the population dynamics. The model is subjected to an independent evaluation in order to assess its descriptive capacity in comparison with previous simulation models, and is shown to constitute an improved tool to describe the seasonal population dynamics of $S$. avenae.
\end{abstract}

(C) 2017 Elsevier B.V. All rights reserved.

\section{Introduction}

The Aphids are ubiquitous crop pests which can cause significant yield losses in economically important cereals (Rautapaa, 1966; Vickerman and Wratten, 1979). At high population densities, these hemipterans convey damage across a range of plant species via: (1) Extraction of nutrients during feeding, (2) production of exudates that provide a suitable substrate for the growth of sooty moulds (Dedryver et al., 2010), and (3) as vectors of plant viruses. Of the approximate 700 plant viruses recognised, almost $50 \%$ of the insect-borne viruses are vectored by aphid species and many of these viruses are responsible for diseases in economically important crops (Katis et al., 2007). Belonging to this group, the grain aphid (Sitobion avenae) is one of the most economically important pests of cereal crops internationally, owing to its tendency to colonise the ear of cereals as soon as it emerges, as well as its efficacy

Abbreviations: SAV4, the new model presented here; SAM7, Carter's (1978) aphid model; SACSIM, previous aphid model by Skirvin et al. (1997).

* Corresponding author at: Room 2.5, Irish Climate Analysis and Research Units (ICARUS), Department of Geography, Laraghbryan House Maynooth University, Ireland.

E-mail addresses: catriona.duffy@nuim.ie (C. Duffy),rowan.fealy@nuim.ie (R. Fealy), Reamonn.Fealy@teagasc.ie (R.M. Fealy). as a vector of the plant virus Barley Yellow Dwarf Virus (BYDV). Due to this species high rate of reproduction, along with its dispersive capacity, outbreaks are common and inherently affected by both climatic and biotic conditions (Kindlmann et al., 2007).

Traditionally, control of aphids and their associated viruses has been achieved via the routine application of chemical controls, irrespective of the level of risk from season to season. This approach has resulted in both negative ecological effects, as well as the emergence of resistance in a number of different aphid species, many of which are considered the most 'problematic pests worldwide' (Foster et al., 2007: 261). The aphid's ability to reproduce parthenogenetically (asexual reproduction without the requirement for fertilisation) serves to ensure that any mutations which are advantageous against chemical controls will be propagated quickly within the population, resulting in pesticide-resistant genotypes, such as those recently discovered in the UK (Dewar, 2014).

Forecasting and warning systems have an important role to play in the control of these pests via Integrated Pest Management (IPM), and complement the relatively recent changes to pesticide regulations in the European Union (European parliament and council of the European Union, 2012). The ability to accurately forecast the abundance of pests such as $S$. avenae on cereal crops in the short term could serve to reduce the prophylactic application of 
aphicides, by providing information to farmers regarding the necessity/timing of chemical applications throughout the season. This type of ecologically-based pest management would serve to reduce both costs and yield losses; as well as reduce the occurrence of unintended negative effects on non-target fauna. On a longer timescale, the application of pest models can serve to provide an indication of potential future pest response to a changing climate, ultimately guiding strategic decision-making and facilitating the modification of crop projections in line with the outcome of pest models.

The model described here is not the first model proposed to describe the population dynamics of an aphid pest species using systems analysis and simulation modelling. Indeed, three of the most economically important aphid pest species have been the subject of such models including Metopolophium dirhodum (the rose-grain aphid) (Zhou et al., 1989), R. padi (Morgan, 2000), S. avenae (Carter, 1985; Carter et al., 1982; Plantegenest et al., 2001; Rabbinge et al., 1979; Skirvin, 1995), as well as the concomitant barley yellow dwarf virus vectored by the two latter species (Kendall et al., 1992; Morgan, 1996; Thackray et al., 2009). These three species have also been incorporated into a single model together, along with their associated predators (Gosselke et al., 2001). To date, these models have been developed over a wide range of countries including the UK, Germany, France and the Netherlands with varying degrees of success.

The work presented here integrates biological data from various studies to develop a new simulation model named SAV4 (Sitobion avenae mark 4) which describes the temperature-dependant development of spring/summer populations of S. avenae. SAV4 has adopted a similar structure to previous aphid simulation models wherein each submodule within the overall simulation model represents a different aspect of the aphid's life cycle (e.g. Carter et al., 1982; Morgan, 2000; Skirvin et al., 1997; Zhou et al., 1989). This model incorporates different components describing the life cycle history of $S$. avenae, including immigration, reproduction, survival, development and morph determination. Each of these submodules interact with each other to facilitate the calculation of phenological and quantitative aphid metrics in response to daily temperature variations. Elements of the model are based on Carters (1978) original model (hereafter referred to as SAM7) and Skirvin's modified version of the same model (hereafter referred to as SACSIM); however the core development submodel has been completely redeveloped, and the equations used for other model components have been updated/improved. The model was programmed in Matlab and can be found in Appendix A and B. Both SAV4 and SACSIM were developed for the UK and were designed to model the dynamics of $S$. avenae in conjunction with predator populations in wheat. The model outlined here utilises literaturederived data from UK sources and resulting empirical relationships to describe the dynamics of $S$. avenae in the absence of biological control/predator factors.

\subsection{Justification for updating previous models}

SAM7 was modified by Skirvin et al. (1997), to incorporate nonlinearity of the primary dataset (from Dean, 1974), as well as to incorporate separate developmental equations for each of the aphid instars (a full description of which can be found in Skirvin et al. (1997)). Skirvin et al. (1997) chose a sigmoidal function (following Stinner et al., 1974) to describe the development of S. avenae, despite the fact that this function is asymptotic to the $\mathrm{x}$-axis at both low and high temperatures (hampering the derivation of both the lower temperature threshold (LT) and the upper temperature threshold (UT)). The Stinner et al. (1974) model utilised in SACSIM also assumes symmetry about the optimum, which is not biologically realistic, and a number of authors (e.g. Kontodimas et al., 2004; Logan et al., 1976; Wagner et al., 1984) have suggested that this model is inaccurate at high temperatures as a result of this symmetry. The current work improves upon the model of Skirvin et al. (1997), by fitting a more suitable nonlinear model to the development data of individual instars of $S$. avenae, in order to better describe the temperature-dependent development of aphid instars. In addition to the improvement of the developmental core of the model, a number of modules describing different facets of the aphid's life cycle have been either updated or redesigned, the details of which are expanded upon within the model description.

Unlike SAM7 and SACSIM, the current model does not take into account the effects of an aphidophagous predator. The omission of predator dynamics from the current model is not intended to suggest their lack of importance, to the contrary, in years when aphid numbers are low, natural enemy activity can be accredited with reducing initial population numbers (Poehling et al., 2007). Their omission in this case is in recognition of the likelihood that any potential regulatory control which could be conveyed upon aphids would be by an entire guild of natural enemies, as opposed to regulation by a lone predatory species. Furthermore, the current model will serve to investigate if the overall population dynamics of $S$. avenae can be captured without the need for data-hungry predator or natural enemy submodules.

\section{Materials and methods}

\subsection{Description of SAV4}

The use of a simulation model enables the simplification of various biological processes down to their component parts, which can in turn be allowed to interact within a larger 'systems' framework (Fig. 1). This simplification of reality facilitates the simulation of complicated aphid dynamics despite the absence of a stable age distribution and the existence of simultaneous overlapping generations. SAV4 assumes that the entire population of $S$. avenae overwinters anholocyclically (in an active form), which allows them to respond immediately to increasing temperatures in the spring, facilitating maturation and reproduction as soon as temperatures are adequate (Bale, 1989). The model is initiated by providing a start date and daily aphid numbers entering the field. Incoming alates are assumed to reproduce as soon as they alight in-field. Reproduction is dependent on temperature, morph and the crop growth stage. The newly born nymphs are firstly identified as either alate or apterous morphs depending on the crop growth stage (GS) and population density, and then begin development in response to temperature on an hourly basis. The nymphs 'age' until they become adult and emigrate (in the case of alates) or they enter a pre-reproductive phase, before themselves becoming reproductively capable (in the case of apterous individuals) and producing new nymphs which will in turn age through the model.

\subsection{Data inputs and outputs}

The model requires a number of data inputs in order to initiate the simulation of the seasonal dynamics of $S$. avenae. These inputs include daily suction trap catches or field counts of aphids (per tiller), as well as the start day of aphid migration. Data describing the daily aerial dynamics of $S$. avenae at the Brooms barn suction trap site in Norfolk were obtained (courtesy of Rothamsted research) from the Rothamsted insect survey (Harrington and Woiwod, 2007). Data collected at this station was used owing to its proximity to the observed field data used in the evaluation below (Section 3.1). The survey is comprised of a collection of fifteen suction traps that primarily samples aphids and has been running since 1964. Minimum and maximum daily temperature values required to drive development within the model were obtained from the 


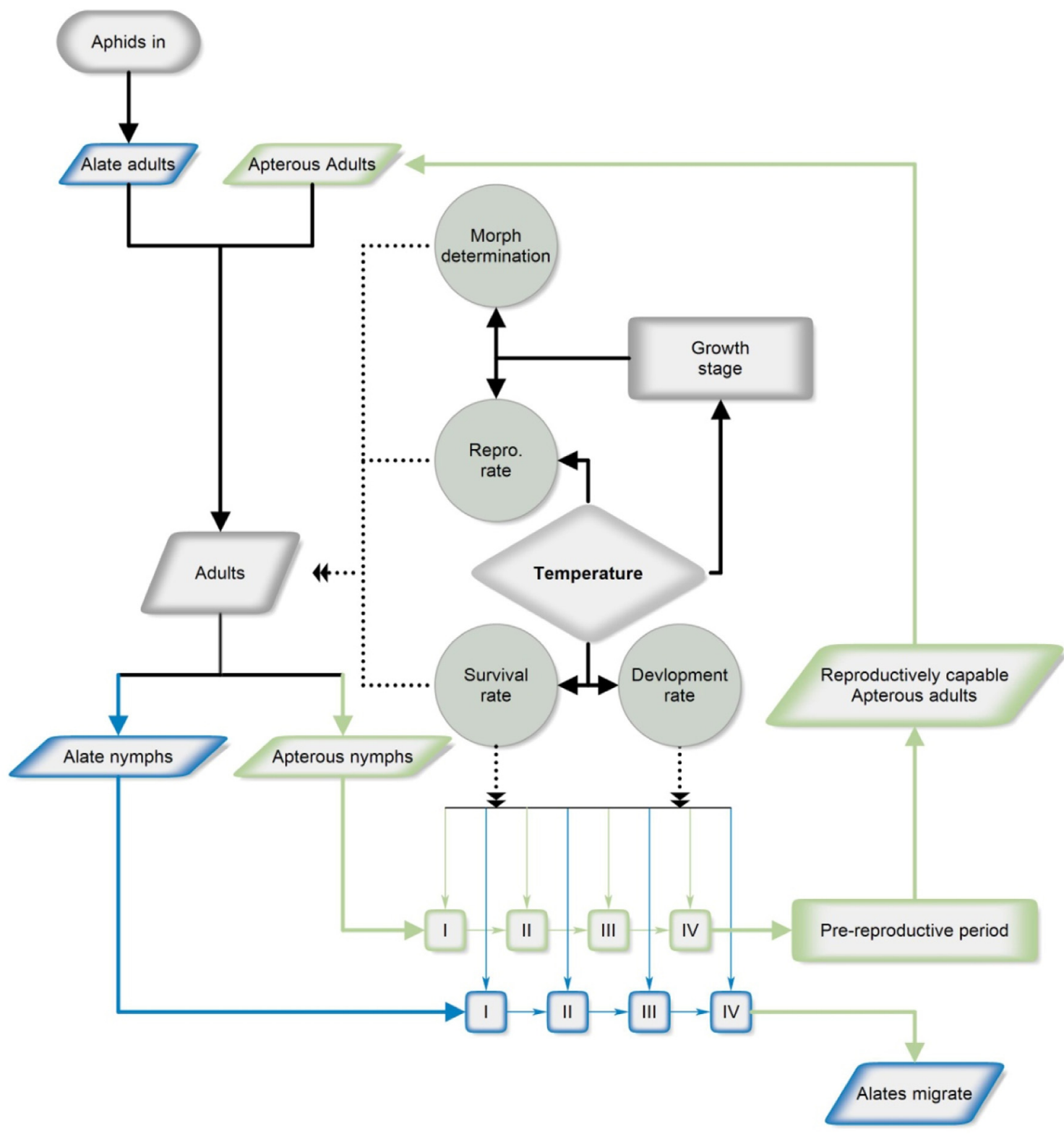

Fig. 1. Process diagram illustrating the physical framework of SAV4.

BADC database (2014) for the local meteorological station used in the final model evaluation (Morley St. Botolph, MIDAS SRC ID:422). The outputs produced by SAV4 include the timing of the peak population numbers (measured by the day of the year (DOY)) as well as the magnitude of the peak aphid number (measured in aphids per tiller).

\subsection{Immigration}

Initial aphid numbers in-field are calculated using daily suction trap catches. The utilisation of suction trap catches to initialise SAV4 incorporates the assumption that aphid numbers in the field can be calculated using these catches; which is supported by field evidence from other aphid species (Harrington and Woiwod, 2007) and other geographic locations (Teulon et al., 2004). The immigration submodel produces the number of aphids in-field, by multiplying the daily catch data of $S$. avenae by two separate factors: a 'deposition factor' and a 'concentration factor'. The former has been calculated based on the aphids mean flight time (Table 1 ) along
Table 1

Number of aphids alighting in field (per hectare) per each individual aphid caught in a suction trap (from Taylor and Palmer, 1972).

\begin{tabular}{llllllll}
\hline \multirow{2}{*}{$\begin{array}{l}\text { Density } \\
\text { Gradient }\end{array}$} & \multicolumn{7}{l}{ Mean Flight Time (Hrs) } \\
\cline { 2 - 8 } & 0.5 & 1 & 2 & 4 & 8 & 12 & 24 \\
\hline 0 & 10315 & 5157 & 2579 & 1289 & 645 & 430 & 215 \\
-0.5 & 1660 & 830 & 415 & 207 & 104 & 69 & 35 \\
-1.0 & 948 & 474 & $* 237$ & 119 & 59 & 40 & 20 \\
-1.5 & 2016 & 1008 & 504 & 252 & 126 & 84 & 42 \\
-2.0 & 10315 & 5157 & 2579 & 1289 & 645 & 430 & 215 \\
\hline
\end{tabular}

a concentration gradient (Taylor and Palmer, 1972) and facilitates an estimation of the rate at which aphids are 'deposited' in-field. As with previous applications of this approach (e.g. Morgan, 2000; Zhou et al., 1989), the flight time was assumed to be 2 hours and the density-height gradient is -1 ; providing a total of 237 aphids (asterisk in Table 1) per hectare for each aphid caught in the suction trap. The latter factor adjustment (the concentration factor), refers to a phenomenon outlined by Carter (1985), wherein the deposition 
factor is found to underestimate the number of aphids in-field by a factor of 40. This factor was also applied in the calculation of initial aphid numbers in-field for the SAV4 simulations. The calculated daily numbers alighting in-field are then used to 'seed' the model by providing the reproducing alate cohort. It is assumed that these individuals have recently moulted and will remain in the crop until they die. The model does not incorporate topographic characteristics; which means that all fields are assumed to be the same. This means that aphid numbers are not modified to allow for field characteristics (slope, soil type, drainage etc), all of which could impact crop growth, and as a result, pest dynamics. This is an area that could be assessed for inclusion in the next iteration of the model presented here.

\subsection{Temperature}

Hourly temperatures were simulated using daily temperature data for two reasons: (1) Owing to the instantaneous rate at which aphids have been shown to respond to temperature (Rabbinge et al., 1979), it was deemed prudent to incorporate hourly timesteps. (2) Additionally, the data used to calibrate the development submodel was based on hourly data (Dean, 1974). The current study utilised a 'WAVE' model after Hoogenboom and Huck (1986); a description of which can be found in its entirety in Reicosky et al. (1989). The day is split into two portions: the first half of the day is modelled using the minimum temperature ( $\mathrm{Tmin}$ ) and the maximum temperature (Tmax) from that day; while the second segment uses the Tmax of the same day in conjunction with the Tmin of the following day. The 'suncycle' function (Begler, 2008) was used in order to simulate the time of sunrise, based on the Julian date and the latitude of the model location. Following Hoogenboom and Huck (1986), the daily Tmin is then set at the simulated time of sunrise, while the Tmax is set at 2 pm daily. The equations comprising the WAVE model simulating the hourly temperature are as follows:

$$
\begin{aligned}
0 & \leq \mathrm{H}<\mathrm{RISE} \text { and } 1400<\mathrm{H} \leq 2400 T(H) \\
& =\operatorname{Tav}+\operatorname{amp}\left(\cos \left(\frac{\pi h}{10+R I S E}\right)\right)
\end{aligned}
$$

$\operatorname{RISE} \leq \mathrm{H} \leq 1400 T(H)=\operatorname{Ta} v-\operatorname{amp}\left(\cos \left(\frac{\pi(H-R I S E)}{14-R I S E}\right)\right)$

where RISE is the time of sunrise in hours ( $24 \mathrm{~h}$ clock) and $\mathrm{T}(\mathrm{H})$ is the temperature at any hour. $\mathrm{H}$ is the time in hours measured on a $24 \mathrm{~h}$ clock. $\mathrm{h}=\mathrm{H}+10$, if $\mathrm{H}<\mathrm{RISE}$ and $\mathrm{h}=14$ if $\mathrm{H}>1400$. Tav and amp, correspond to the average temperature and amplitude of the daily temperature cycle, and are defined as: $\operatorname{Tav}=(\operatorname{Tmin}+\operatorname{Tmax}) / 2$, and $\operatorname{amp}=(\operatorname{Tmax}-\mathrm{Tmin}) / 2$.

\subsection{Reproduction}

Reproduction is dependent on the temperature experienced by the aphid, the morph of the aphid, as well as the crop GS. Apterous individuals have previously been found to exhibit higher fecundity levels (Ankersmit and Dijkman, 1983; Wratten, 1977) than that of alates, and for that reason, each morph is treated separately. The submodel consists of two linear functions fitted to the data of Dean (1974) for apterous individuals and two functions for alates (Fig. 2). The first equation to describe the relationship between reproduction and temperature was fitted to Dean (1974) data from zero development at $3^{\circ} \mathrm{C}(\mathrm{LT})$ to maximum development at $20^{\circ} \mathrm{C}$; while the second was fitted in agreement with Skirvin's approach (between 20 and $30^{\circ} \mathrm{C}$ ). The reproductive LT where zero reproduction occurs has been amended from that utilised by Skirvin (1995). This modification of the reproductive LT is in line with results summarised by Williams and Wratten (1987), who stated that the
Table 2

Fecundity parameters for both morphs of S. avenae in response to temperature.

\begin{tabular}{llll}
\hline Morph & Temperature Range & $\beta_{0}$ & $\beta_{1}$ \\
\hline Apterous & $3-20^{\circ} \mathrm{C}$ & -0.3766 & 0.1772 \\
Apterous & $20-30^{\circ} \mathrm{C}$ & 9.1917 & -0.3050 \\
Alate & $3-20^{\circ} \mathrm{C}$ & -0.3653 & 0.1218 \\
Alate & $20-30^{\circ} \mathrm{C}$ & 6.2100 & -0.2070 \\
\hline
\end{tabular}

temperature-reproduction relationship was well described when temperatures above $3^{\circ} \mathrm{C}$ were used. For that reason, a reproductive LT of $3{ }^{\circ} \mathrm{C}$ was included and the corresponding linear functions to describe apterous rates of reproduction below $20^{\circ} \mathrm{C}$ were updated accordingly (Fig. 2(a)). The form of the linear function is described in Eq. (3), where y is the dependent variable (number of nymphs), $\beta_{0}$ is the intercept, $\beta_{1}$ is the slope and $x$ is the independent variable (in this case, temperature). The parameters of each separate regression are provided in Table 2.

$y=\beta_{0}+\beta_{1} x$

In the case of alate reproduction, Wratten's data (1977) was used to model the reproductive capacity of alates at $20^{\circ} \mathrm{C}$. Two linear functions were then fit to the data (Table 2): One between the reproductive LT of $3^{\circ} \mathrm{C}$ and the $\mathrm{T}^{\mathrm{opt}}$ of $20^{\circ} \mathrm{C}$; and the second between the Topt and UT of $30^{\circ} \mathrm{C}$ (Fig. 2(b)). This step is justified by the linear relationship between reproduction and temperature described by Williams and Wratten (1987). Wratten's (1977) data was utilised in conjunction with an assumed 20 day adult survival period to calculate the mean daily nymphs produced over this time period (after Dean, 1974 and Wratten, 1977). This submodel assumes that alates that alight in-field are immediately reproductively capable, while all apterous individuals must pass through a pre-reproductive delay before reproducing. Alate individuals that mature within the model are also assumed to emigrate as soon as they reach maturity. As a result, the only reproducing alates in the model are those individuals who are deposited in-field.

The variability of the reproductive rate of $S$. avenae in response to its host plant was highlighted by Watt (1979). As with previous models (e.g. Carter, 1978; Skirvin, 1995), an increase in reproductive rate (multiplied by 1.6) was applied at ear emergence and before the milky-ripe stage (GS 59-73). This multiplicative factor is in line with the findings of Watt (1979), wherein aphids were reported to be 1.6 times more fecund on the ears than at other plant growth stages. The reproductive rate drops to zero after GS 80; as the crop is no longer suitable for aphid reproduction (Watt, 1979). The reproductive data utilised here to produce reproductive rates of both morphs were not available at hourly intervals, but rather on a daily timestep (Dean, 1974; Wratten, 1977). As a result, nymph production was simulated on a daily, rather than hourly timescale in order to minimise the potential for error, as well as the assumptions required to disaggregate the daily reproductive rate data over a $24 \mathrm{~h}$ period.

\subsection{Morph determination}

The morph that each aphid develops into is determined at birth. All nymphs produced by both alate and apterous parents are summed before their morph (alate or apterous) is determined. The morph is dependent on both the crop developmental stage and the density of aphids at that particular timestep. This finding has been widely iterated throughout the literature, citing increases in alate production concurrently with the deterioration of the host plant and crowding (e.g. Sutherland, 1969; Watt and Dixon, 1981). The equation used to describe the percentage of nymphs that become alates is:

Percentagealates $=2.603 \times$ Aphiddensity $+0.847 \times$ GS $-27.189(4)$ 

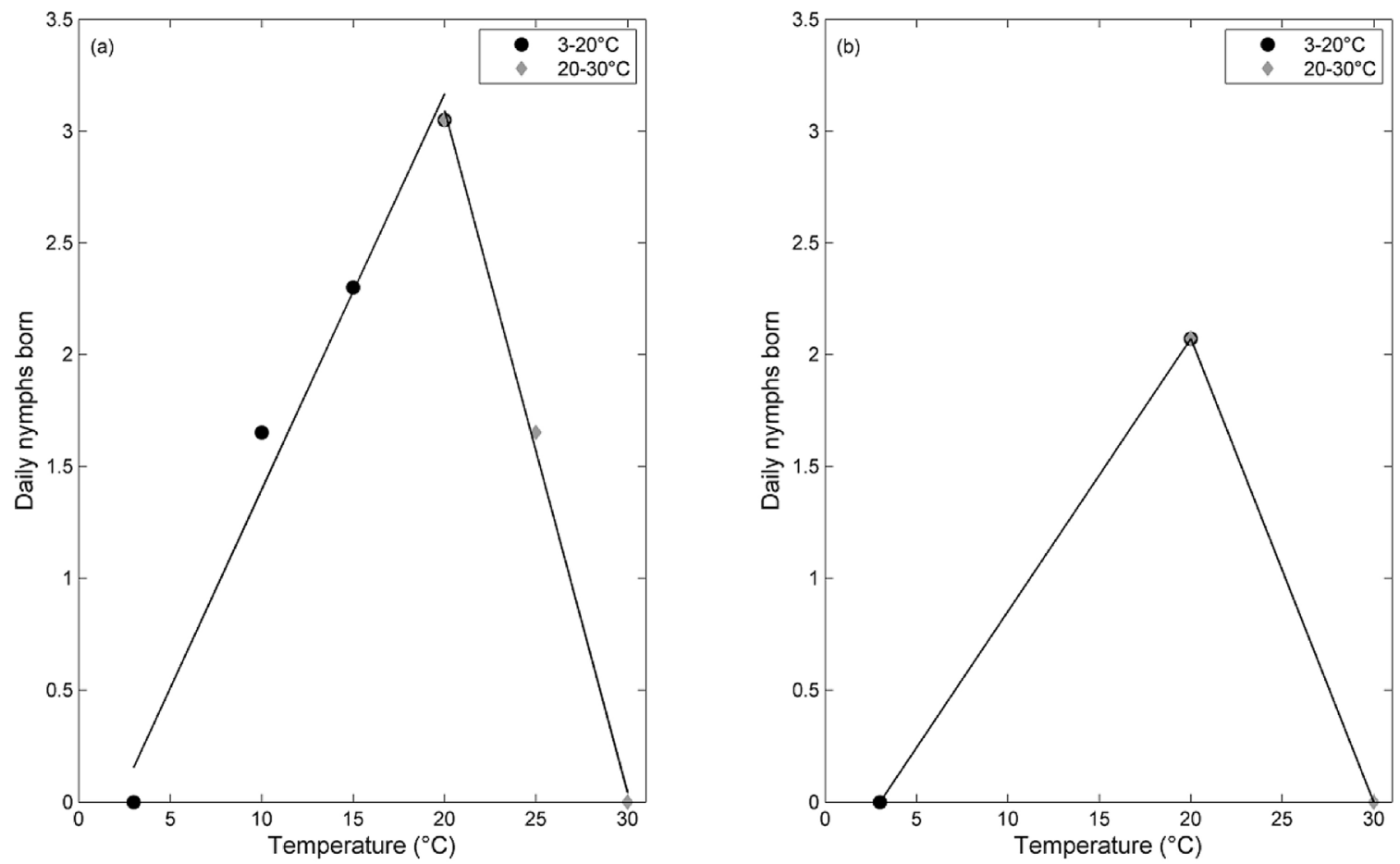

Fig. 2. Fecundity of apterous (a) and alate (b) S. avenae in response to temperature (Source: Dean, 1974).

This equation is adopted from Carter et al. (1982), and has been previously applied elsewhere (e.g. Skirvin et al., 1997; Zhou et al., 1989). Eq. (4) relates the proportion of nymphs that develop into alates, to the crop GS and the density of aphids per tiller on the crop at the birth-time of nymphs.

\subsection{Nymph and adult survival}

Survival is treated separately for nymphs and adults in the current model. Due to the lack of detailed survival data for $S$. avenae, a pragmatic approach to introducing mortality to the system was utilised. Mean mortality of immature stages in the temperature range of $10-30^{\circ} \mathrm{C}$ is available from Dean (1974) and is generally low across the instars. This finding is supported by Williams and Wratten (1987) analysis which reported nymphal survival means of $97 \%$. Using this data, a survival probabi lity is applied daily to the nymphs in the system. As new aphids are 'born' on a daily basis the survival probability is applied. This is accomplished by multiplying the survival probability by the number of nymphs in the system daily, the result of which is then subtracted from the overall number of nymphs. The probability of a nymph surviving is adjusted depending on the amount of development which has taken place over the daily timestep. This adjustment is incorporated owing to the fact that the length of the instar changes, depending on the temperature experienced. The equation used is:

Survival $=I^{\frac{H h}{H i}}$

Where, I is the temperature-dependent proportion of nymphs surviving to complete the instar; Hh is the amount of development which took place in the timestep and $\mathrm{Hi}$ is the length of the instar (i.e. 1). The method for calculating I has been adjusted for simplicity and is calculated by fitting an asymptotic regression (Eq. (6)/Fig. 3) to the data of Dean (1974), the coefficients of which are described in Table 3. The standard error of the regression (the S value) is 2.28969 . The $\mathrm{S}$ value (the average distance that the observed values fall from the regression line) was chosen as the fit statistic as opposed to the $R_{2}$, due to the fact that the $R_{2}$ is based on an assumption of linear-
Table 3

Coefficients used in describing nymphal survival in response to temperature.

\begin{tabular}{ll}
\hline Coefficient & Value \\
\hline$\Theta_{1}$ & 94.4449 \\
$\Theta_{2}$ & $3.3221 \mathrm{e}-008$ \\
$\Theta_{3}$ & -0.7256 \\
\hline
\end{tabular}

ity in the model. The $\mathrm{S}$ value uses the same units as the response variable and smaller values are considered better, as they are found closer to the fitted line.

Nymphal survival $(I)=\Theta_{1}-\Theta_{2} \times \exp \left(-\Theta_{3} \times \operatorname{Temp}(\mathrm{I})\right)$

In order to account for the effect of plant GS on nymphal survival, the proportion of nymphs surviving past GS 73 was reduced in accordance with the findings of Watt (1979). A fixed proportion after this stage of 0.45 (Watt, 1979) was selected on the assumption that $S$. avenae's preference for the ears of the crop would be predominant.

For adult survival, a constant longevity of 20 days was adopted from the moment the aphid becomes a reproductively capable adult. This assumption is based on three separate lines of reasoning: Firstly, according to Dean (1974) experimentation, the mean adult life span across the temperature range $10-25^{\circ} \mathrm{C}$ is 20 days. Secondly, Dean (1973) also found that adult S. avenae survived a mean of 20 days when reared at a constant temperature. Finally, according to Wratten (1977), after 20 days adult aphid survival and reproduction are extremely low. For these reasons, adult longevity was limited at 20 days. When adults reached this age within the model, they were removed from the model population.

\subsection{Development}

The relationship between rate of development and temperature for each of the instars of S. avenae is illustrated in Fig. 4. Within SAV4, each of the instar's developmental relationship with temperature is described separately, owing to the fact that Dean (1974) 


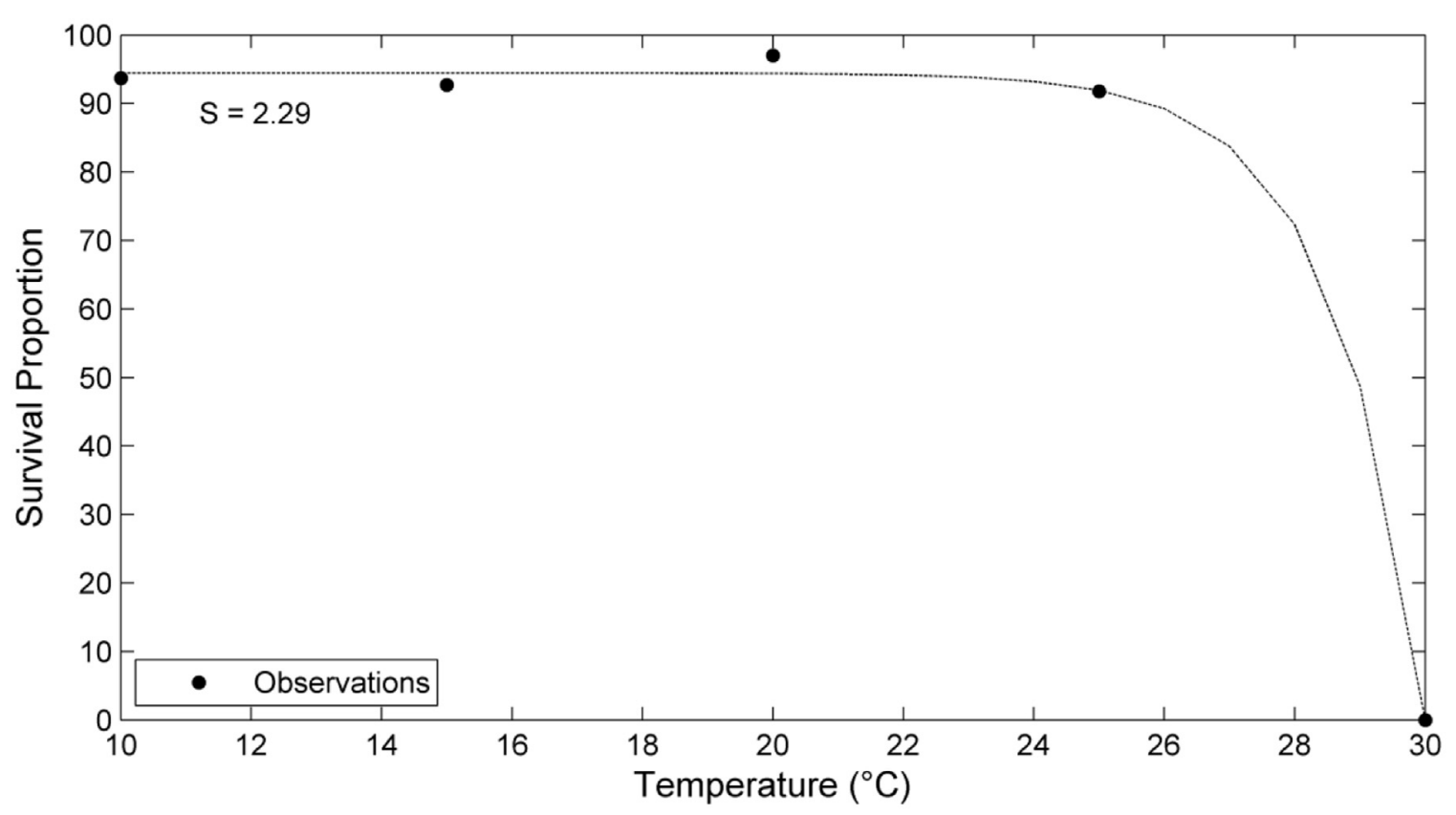

Fig. 3. Proportion of nymphal survival in response to temperature (After: Dean (1974)).

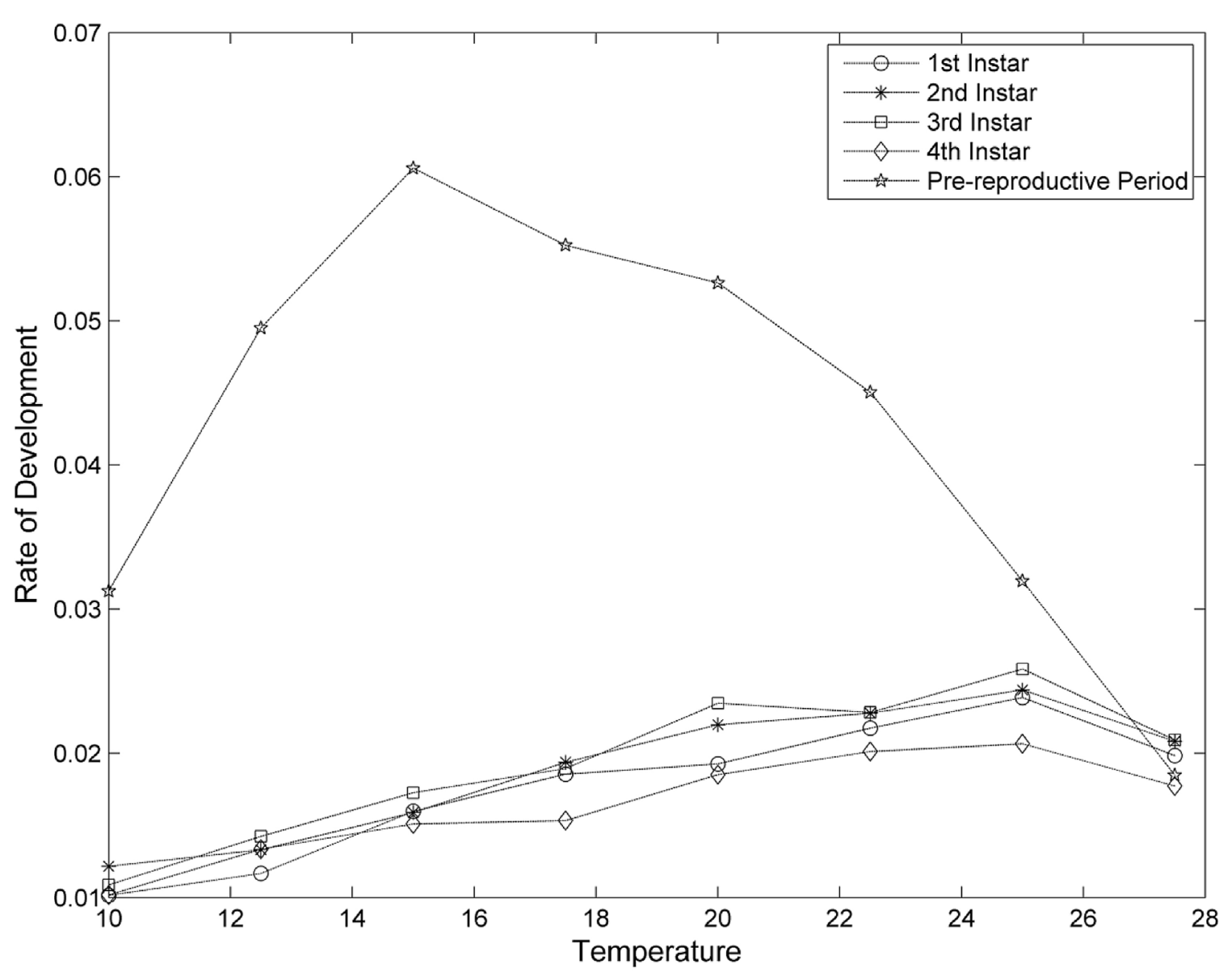

Fig. 4. Rate of development in S. avenae in response to temperature across all developmental stages.

reported data for each of the individual developmental stages independently (Table 4). Using a criteria-led approach (Johnson and Omland, 2004), a nonlinear function hereafter referred to as the 'Lactin model' (Lactin et al., 1995) was selected to quantify the rate of development in the spring/summer population dynamics of $S$. avenae (Eq. (7)). The Lactin equation is

$r(T)=e^{\rho t}-e^{\left[\rho \operatorname{Tmax}-\frac{(\operatorname{Tmax}-T)}{\Delta}\right]}+\lambda$ 
Table 4

Duration (hours) of temperature-dependent development in S. avenae with associated errors in brackets (Dean, 1974).

\begin{tabular}{lllll}
\hline Temperature $\left({ }^{\circ} \mathrm{C}\right)$ & 1st Instar & 2nd Instar & 3rd Instar & 4th Instar \\
\hline 10.0 & $98.5( \pm 4.7)$ & $82.2( \pm 1.7)$ & $91.9( \pm 1.9)$ & $98.2( \pm 1.1)$ \\
12.5 & $85.7( \pm 1.4)$ & $75.1( \pm 1.3)$ & $70.2( \pm 1.4)$ & $74.9( \pm 1.0)$ \\
15.0 & $62.6( \pm 2.4)$ & $62.9( \pm 1.5)$ & $57.9( \pm 2.0)$ & $66.2( \pm 1.0)$ \\
17.5 & $53.9( \pm 0.7)$ & $51.6( \pm 0.8)$ & $52.8( \pm 1.3)$ & $65.2( \pm 0.9)$ \\
20.0 & $51.9( \pm 0.9)$ & $45.5( \pm 0.7)$ & $42.6( \pm 0.8)$ & $54.0( \pm 0.8)$ \\
22.5 & $46.0( \pm 1.5)$ & $43.9( \pm 2.1)$ & $43.8( \pm 1.6)$ & $49.7( \pm 0.9)$ \\
25.0 & $41.9( \pm 0.8)$ & $41.0( \pm 1.1)$ & $38.7( \pm 1.1)$ & $48.4( \pm 1.2)$ \\
27.5 & $50.4( \pm 1.2)$ & $48.0( \pm 1.3)$ & $47.8( \pm 1.4)$ & $56.4( \pm 1.0)$ \\
\hline
\end{tabular}

Table 5

Lactin-derived LT and UT for four separate instars of S. avenae.

\begin{tabular}{lll}
\hline Instar & LT & UT \\
\hline 1st & $0.5^{\circ} \mathrm{C}$ & $29.3^{\circ} \mathrm{C}$ \\
2nd & $-1.7^{\circ} \mathrm{C}$ & $30.1^{\circ} \mathrm{C}$ \\
3rd & $0.3^{\circ} \mathrm{C}$ & $29.8^{\circ} \mathrm{C}$ \\
4th & $-4.0^{\circ} \mathrm{C}$ & $29.8^{\circ} \mathrm{C}$ \\
\hline
\end{tabular}

Where $\mathrm{T}$ is temperature, $\mathrm{r}(\mathrm{T})$ is the rate of development at temperature T, Tmax is the supraoptimal temperature at which $\mathrm{r}(\mathrm{T})=\lambda . \Delta$ and $\rho$ are parameters to be estimated (the range of temperatures between Tmax and the temperature at which $\mathrm{r}(\mathrm{T})$ is maximum, and the acceleration of the function from the LT to the UT respectively).

The selection of an appropriate model took cognisance of the biological realism of the parameters, as well as the fit of the data to the parameterised function as in Kontodimas et al. (2004). Other criteria considered in the selection included the minimisation of the number of parameters to be estimated, as well as the requirement of the function to simulate all the critical temperature thresholds of interest. A number of different nonlinear functions were tested for their adherence to predefined criteria, ultimately identifying the chosen Lactin model as the optimal nonlinear function for describing the temperature-dependent rate of development in S. avenae. The selection of this model not only takes account of the nonlinearity of the data, but it also employs a more biologically realistic function and parameters than utilised in previous models (e.g. Carter et al., 1982; Skirvin et al., 1997). While many available functions are based entirely in empiricism, the Lactin model includes parameters which can be interpreted biologically (e.g. $\rho$ as a rate increase). Each of the curves presented in Fig. 5 were fitted by iterative nonlinear regression (Minitab version 16.1.1) based on the Marquardt algorithm which is informed by the partial derivatives of the dependent variable with respect to each parameter. This approach involves iterative alterations to the parameter values in an effort to reduce the sum of squared errors between the data points (Dean, 1974) and the function (i.e. the algorithm converges on the set of parameters which minimise the sum of the squared residuals). The derived LT and UT for each of the instars of S. avenae are displayed in Table 5.

Development is quantified by summing the instantaneous fractions of development (rT) in response to hourly temperature using the Lactin function (Lactin et al., 1995) parameterised for each individual instar as described above. This quantification of temperature-mediated development is calculated within the model array, facilitating the 'aging' of newly born nymphs daily.

Growth rates have been suggested to decrease in later instars in $S$. avenae, as resources are allocated to embryo development (Newton and Dixon, 1990a, 1990b). This is particularly the case with fourth instar nymphs that are destined to become alate adults (due to the formation of wings). As a result, development in 4th instar alate nymphs takes longer than that of an apterous 4 th instar nymph. The original SAM7 model proposed that the additional time can be quantified as 1.5 times that of the developmental time of
Table 6

Polynomial coefficients used in the calculation of the pre-reproductive period of $S$. avenae.

\begin{tabular}{ll}
\hline Coefficient & Value \\
\hline$P_{1}$ & -0.1688 \\
$P_{2}$ & 0.0327 \\
$P_{3}$ & -0.0014 \\
$P_{4}$ & 0.000019 \\
\hline
\end{tabular}

apterous individuals. This proposition is based on work on a different species, however data which distinguishes between morphs of S. avenae (Lykouressis, 1985) suggest that in this species, the 4th instar also exhibits a longer developmental time in the alate form. As a result, the assumption that 4 th instar stage in the alatiform nymphs takes 1.5 times longer than their apterous counterparts to complete development was also applied here. This longer developmental time was incorporated into SAV4 by calculating the number of hours required for 4th instar apterous nymphs to complete development in response to hourly temperatures and then multiplying that number of hours by 1.5 to produce the 4 th instar alate development time. All alates were assumed to emigrate as soon as their 4th instar was completed, while apterous individuals were assumed to enter a pre-reproductive stage before producing offspring themselves.

\subsection{Pre-reproductive period}

The development rates describing the pre-reproductive period for $S$. avenae was not of a similar shape to the preceding four instars (Fig. 4). As a result, the Lactin model was not a suitable function to describe the pre-reproductive period that apterous individuals pass through before they become reproductively capable adults. A cubic polynomial (Fig. 6) was found to describe this relatively short lived stage in apterous individuals; with an $\mathrm{R}^{2}$ of $98.6 \%$ and is of the form:

$p(x)=P_{1} x^{3}+P_{2} x^{2}+P_{3} x+P_{4}$

Where $\mathrm{p}(\mathrm{x})$ is the pre-reproductive rate, $\mathrm{x}$ is temperature and $\mathrm{P}_{1}$, $P_{2}, P_{3}$ and $P_{4}$ are coefficients to be estimated (Table 6). This polynomial function was treated in the same fashion as the Lactin model and was used to accumulate developmental time in response to temperature for the pre-reproductive period. Fig. 6 illustrates the derived temperature-rate relationship using the above polynomial, while the coefficients of the regression are represented in Table 6.

\subsection{Crop growth}

Cereal crop development is driven by temperature and quantified as growth stages (GS) in accordance with the Zadoks growth scale (ZGS) (Zadoks et al., 1974). The 'ZGS' function within SAV4 utilises a degree-day approach to calculate the crop growth stage, based on the accumulation of daily temperatures above a cropspecific LT. Degree-days provide a measure of the thermal energy available for crop development and are calculated by subtracting the crop LT from the average daily temperature; each degree above the LT represents a degree-day which are then accumulated in time. This module of SAV4 can be re-parameterised to facilitate the production of GS for any cereal crop, as long as GS observational data and daily temperatures over the course of crop development are available.

In order to facilitate an assessment of the current model in comparison with previous models, the GS module was calibrated with observed GS data from Norfolk used in the original SAM7 model (Carter et al., 1982). Weekly winter wheat GS observations from 1976 to 1978 were regressed against accumulated heat units (Cumulated Degree Days) using a cubic polynomial as in Eq. (9), 

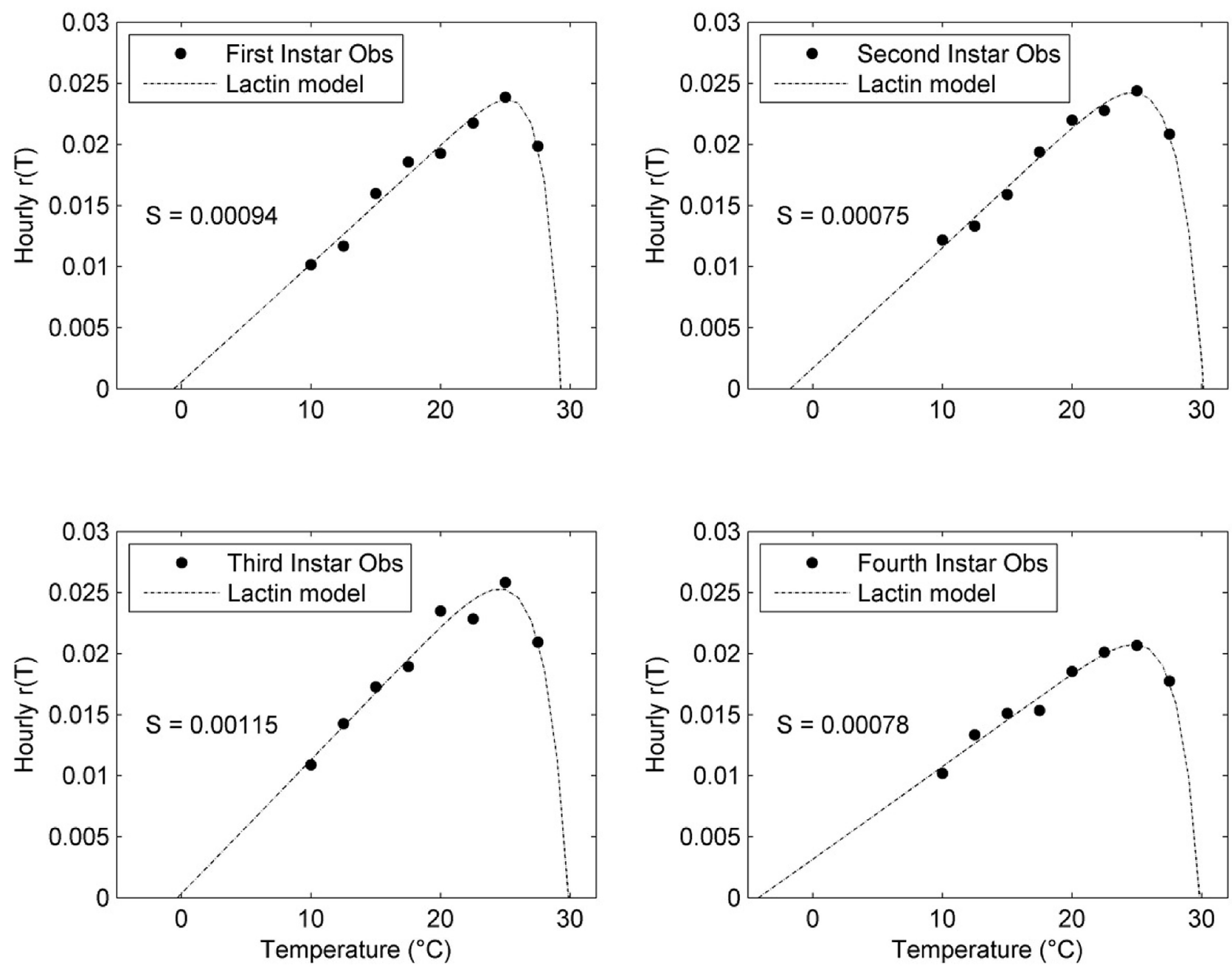

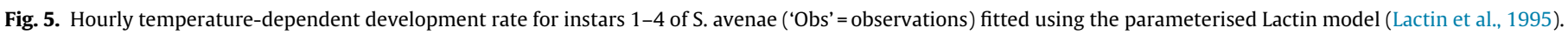

Table 7

Polynomial coefficients utilised to describe the relationship between DD and crop GS.

\begin{tabular}{lllll}
\hline $\mathrm{P}_{1}$ & $\mathrm{P}_{2}$ & $\mathrm{P}_{3}$ & $\mathrm{P}_{4}$ & $\mathrm{R}_{2}$ \\
\hline 0.0000003 & 0.0006549 & 0.4506468 & -12.5395257 & $96 \%$ \\
\hline
\end{tabular}

where $\mathrm{x}$ is the CDD and $\mathrm{P}_{1}, \mathrm{P}_{2}, \mathrm{P}_{3}$ and $\mathrm{P}_{4}$ are coefficients to be estimated. Each of these coefficients are displayed in Table 7. This constitutes a modification to the original approach used in Carter et al. (1982) and Skirvin et al. (1997) (described in Eq. (10)) in that multiple years of data are used to calibrate the crop model, as opposed to a single year (1977).

Using daily temperature data for Norfolk from 1976 to 1978 , the crop growth stage was calculated on a daily timestep within the model and is illustrated in Fig. 7, along with simulated GS output from SAM7 (for comparison) and observed GS data (each set of data is plotted against the DOY). As described in Section 2.2, the temperature data utilised was from the local meteorological station in Norfolk.

$G S=p(x)=P_{1} x^{3}+P_{2} x^{2}+P_{3} x+P_{4}$

$G S=(0.173 \times x)-\left(0.000125 \times x^{2}\right)+26.336$

\section{Results}

\subsection{Model evaluation}

The evaluation of SAV4 has two main components: Firstly, to investigate how well it performs in relation to the field observations and secondly, to test how well the model performs in comparison with previous models. The principle evaluation criterion concerned with assessing SAV4's ability to reproduce observed behaviour in real aphid populations, is achieved by comparing model-derived aphid measurements with field data, and assessing how closely they correspond. The aphid measurements or metrics of interest are (1) peak numbers of aphids per tiller (PN) and (2) the timing of the peak numbers (PD). These metrics were chosen for two reasons: Firstly, they are the same aphid metrics used in previous $S$. avenae models, providing the necessary data for an independent evaluation. Secondly, the use of these metrics also facilitates a direct comparison between the current model and previous models (SAM7 and SACSIM), allowing for the second component of SAV4'S evaluation: an assessment of SAV4's performance in relation to the previous two models.

Observational data was obtained from two different published sources: (1) the original evaluation of SAM7 in Norfolk (Carter et al., 1982) and (2) peak aphid metrics from 1979 to 1984 (Entwistle and Dixon, 1986). Three years of seasonal aphid observations from 1976 to 1978 in Norfolk were chosen for the primary evaluation 


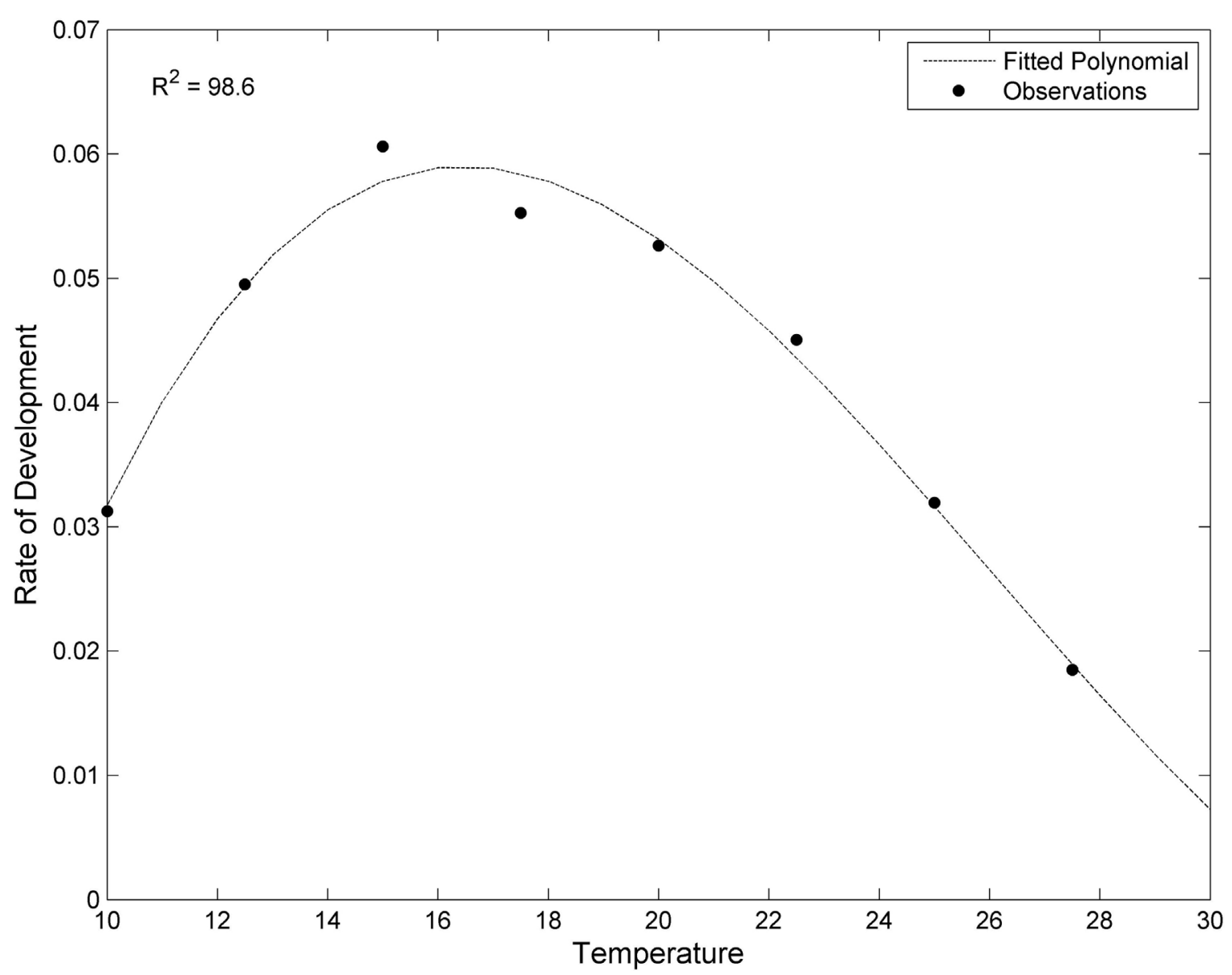

Fig. 6. Pre-reproductive rate of development in apterous S. avenae in response to temperature (Data Source: Dean, 1974).
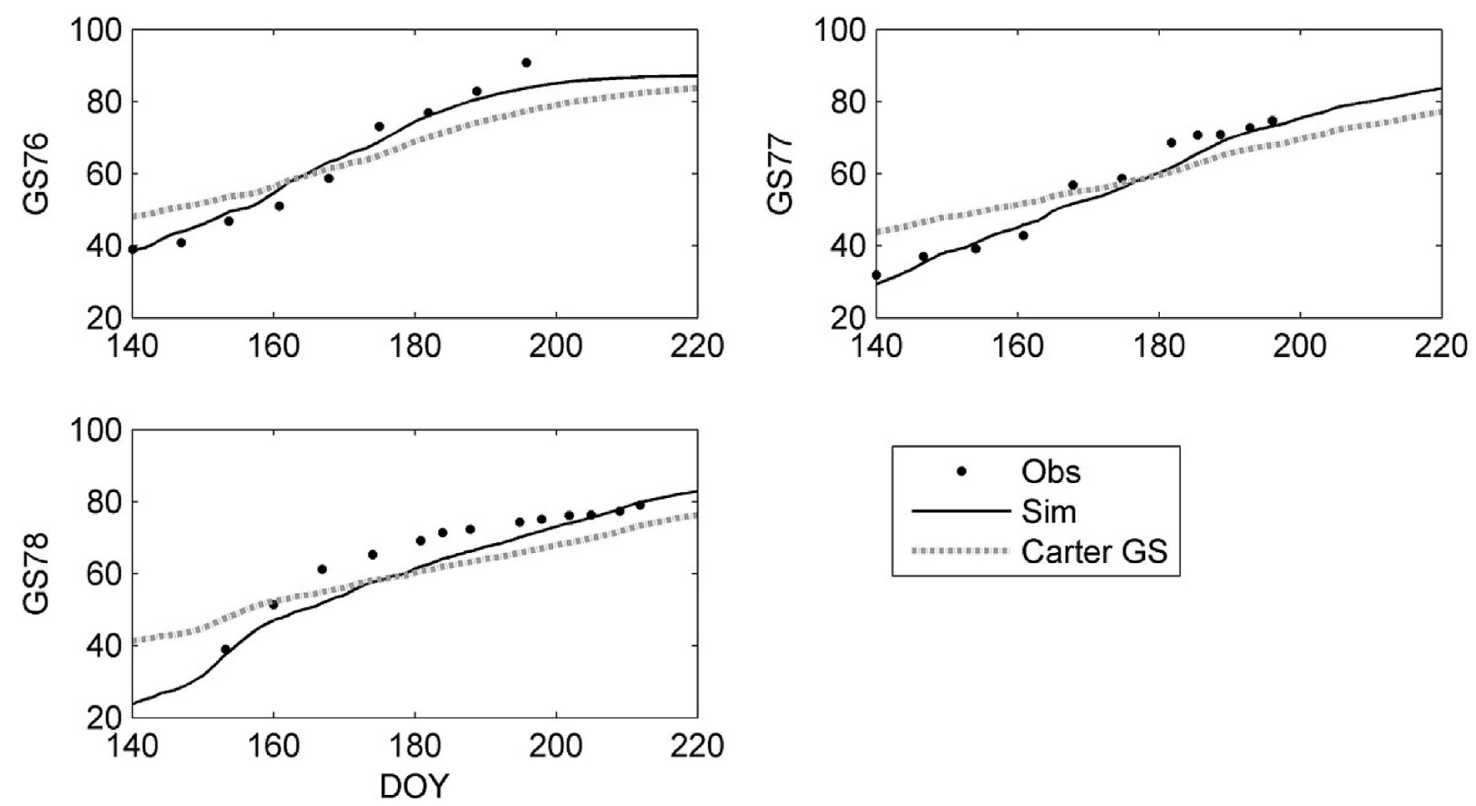

Fig. 7. Comparison of SAV4 polynomial (Eq. (9)) (Sim), Carters GS model (Carter GS) (Eq. (10)) and GS observations (Obs) from 1976 to 1980 inclusive.

and compared with the daily output from SAV4, SAM7 and SACSIM (Figs. 8-10). Two separate fields of seasonal observations were available from 1976 and 1977, while observations for only one field were available for 1978. As a secondary, high level assessment of the model's ability to simulate aphid dynamics, additional peak aphid data from 1976 to 1984 (Entwistle and Dixon, 1986) were also obtained and compared with outputs from SAV4. 


\subsubsection{Evaluation: 1976}

Two fields of observations were used for 1976, both of which were cultivar (cv.) Maris Huntsman (MH). Fig. 8 illustrates the outputs from SAV4 on a daily timestep, along with the field observations and the original output from SAM7 for 1976 for two fields. In addition, Fig. 8(a) also illustrates the output from SACSIM for 1976 (Skirvin et al., 1997), the sole year used in its original evaluation. Carters reduced-predation simulation output was chosen to compare against the current model, as it was considered the most directly comparable to SAV4.

SAV4 performed well in comparison with SAM7 in both field $\mathrm{a}$ and $\mathrm{b}$ (Fig. 8). The timing of the peak was simulated to within 3 days of the observed, in comparison with a 5-day difference produced by SAM7. The simulated PD produced by SACSIM was closer to the observed PD than SAV4, by one day. Within both fields, the magnitude of the observed peak was overestimated by all the models. However, SAV4 overestimated the peak by the smallest amount (an average of just 6 aphids/tiller), constituting a significant improvement in predictive capacity when compared with SAM7 and SACSIM (where the PN was overestimated by an average 30 aphids/tiller) (Table 8).

\subsubsection{Evaluation: 1977}

Once again, two fields were used in the analysis for 1977, where overall SAV4 constituted an improvement on the previous SAM7 model. Fig. 9 illustrates the output from two different fields of winter wheat (cv. Maris Freeman (MF) and cv. MH). In both cases, the peak day was accurately described by SAV4, while SAM7's estima- tion was late by two days (Table 8). SAV4 provided a better fit to the observed PN in field a, while SAM7's prediction of the magnitude was closer to the observed than SAV4 in field $b$.

\subsubsection{Evaluation: 1978}

Only one field was utilised in the analysis for 1978 in Norwich (cv. MH), where both SAV4 and SAM7 produced earlier PD's in comparison with the observations (15 days and 6 days respectively) (Table 8). Both models produced higher number of aphids than observed in the field (Fig. 10). SAV4 calculated the magnitude of the population more accurately than SAM7, with a difference of 8.6 aphids/tiller between modelled and observed (compared with a difference of 11.6 for SAM7). The general shape of the population progression was reproduced by SAV4, with the exception of the initial population rise (SAV4 predicted a higher and more rapid population increase than observed in-field). It is worth noting that the number of aphids in the spring migration was small this season, which partly explains why the observed numbers are low compared with previous years.

\subsubsection{Evaluation: $1979-1984$}

The final step in the evaluation of SAV4, was to run the model for an additional six years, in order to provide a comparison against the remaining peak aphid data from 1979 to 1984 (Entwistle and Dixon, 1986). Each of the final peak metric outputs from SAV4 are illustrated in Fig. 11, along with the observed peak days and peak numbers in Norwich from 1976 to 1984 (Table 9). Peak outputs from SAM7 are also provided where available (1976-1980) to
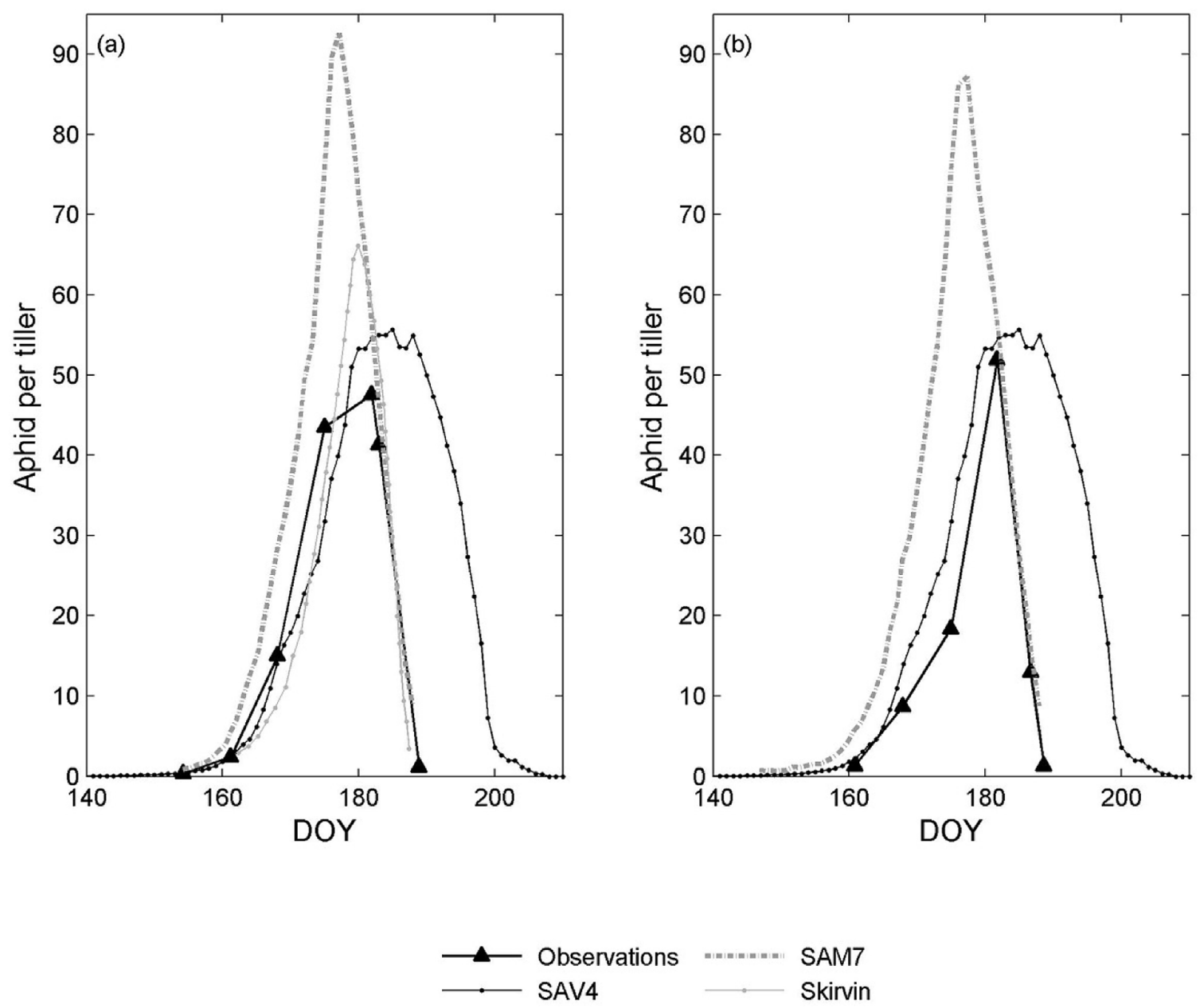

Fig. 8. Comparison of in-field aphid observations from 1976 in two fields (cv. MH) in Norwich, with output from SAV4 and SAM7. 

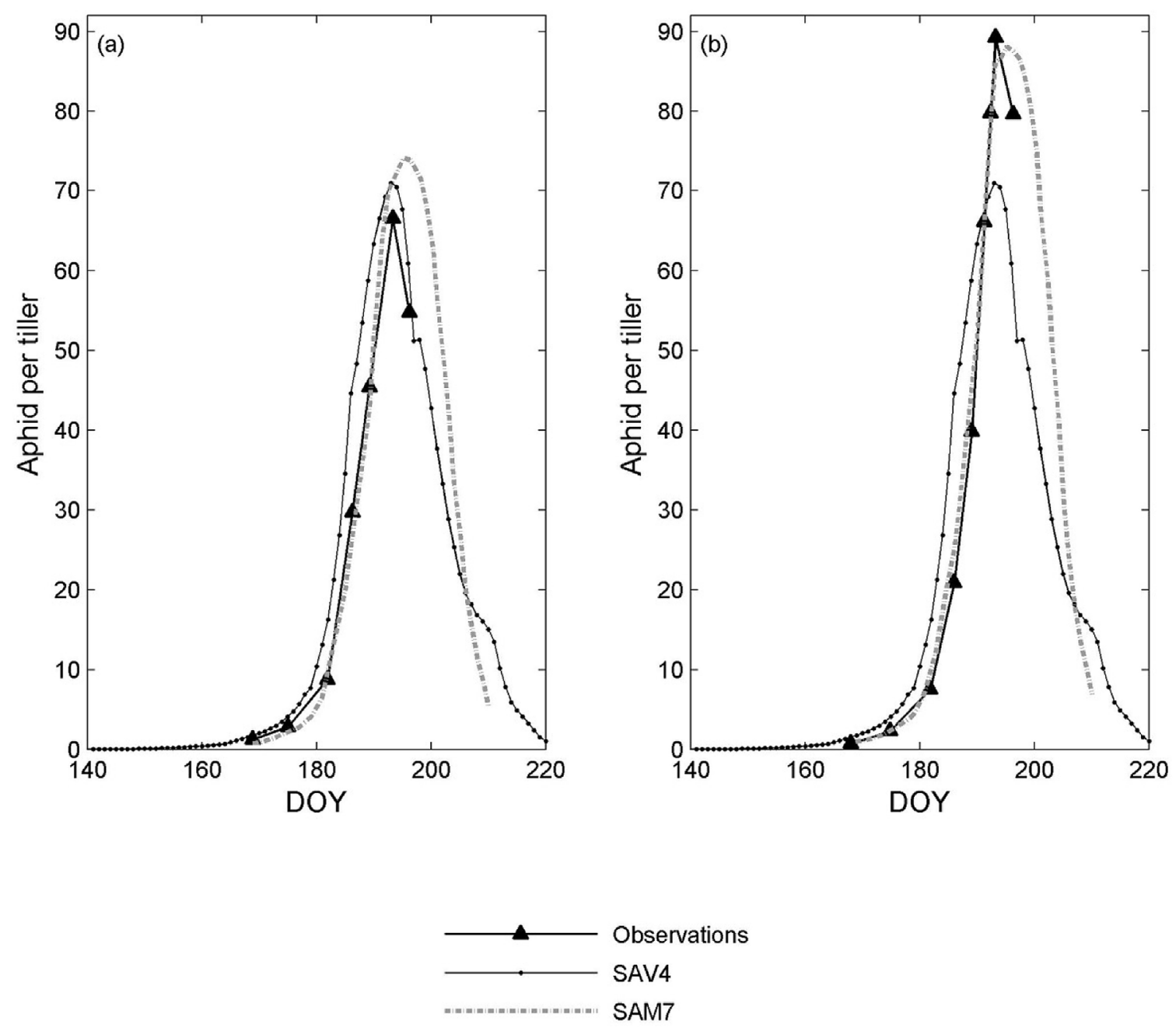

Fig. 9. Comparison of in-field aphid observations from 1977 in two fields in Norwich ((a):cv.MF, (b):cv.MH), with output from SAV4 and SAM7.

Table 8

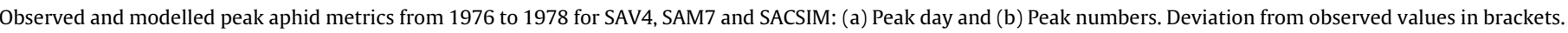

\begin{tabular}{|c|c|c|c|c|}
\hline (a) Year & Obs Peak day & SAV4 Peak day & SAM7 Peak day & SACSIM Peak day \\
\hline 1976a & 182 & $185(+3)$ & $177(-5)$ & $180(-2)$ \\
\hline $1976 b$ & 182 & $185(+3)$ & $177(-5)$ & - \\
\hline $1977 a$ & 193 & $193(0)$ & $195(+2)$ & - \\
\hline 1977b & 193 & $193(0)$ & $195(+2)$ & - \\
\hline 1978 & $205 / 215$ & $200(-15)$ & $209(-6)$ & - \\
\hline (b) Year & Obs Peak num. & SAV4 Peak num. & SAM7 Peak num. & SACSIM Peak num. \\
\hline 1976a & 47.5 & $55.7(+8.2)$ & $92.6(+45.1)$ & $66.2(+18.7)$ \\
\hline $1976 b$ & 51.9 & $55.7(+3.8)$ & $87.2(+35.3)$ & - \\
\hline $1977 a$ & 66.5 & $71.0(+4.5)$ & $74.1(+7.6)$ & - \\
\hline 1977b & 89.3 & $71.0(-18.3)$ & $88.1(-1.1)$ & - \\
\hline 1978 & 5.0 & $13.6(+8.6)$ & $16.6(+11.6)$ & - \\
\hline
\end{tabular}

Table 9

Modelled and observed (Obs) (a) peak day (measured in DOY) and (b) peak number of aphids annually from 1976 to 1984 in Norwich.

\begin{tabular}{|c|c|c|c|c|c|c|}
\hline Year & Obs peak day & SAV4 peak day & SAM7 peak day & Obs Peak num & SAV4 peak num & SAM7 peak num \\
\hline 1976 & 182 & 185 & 177 & 47.70 & 55.68 & 92.60 \\
\hline 1977 & 193 & 193 & 196 & 67.10 & 71.0 & 87.0 \\
\hline 1978 & 216 & 200 & 209 & 5.04 & 13.57 & 16.60 \\
\hline 1979 & 208 & 208 & 207 & 5.46 & 0.38 & 1.89 \\
\hline 1980 & 204 & 191 & 185 & 39.10 & 111.82 & 106.50 \\
\hline 1981 & 182 & 181 & $\mathrm{n} / \mathrm{a}$ & 1.84 & 128.08 & $\mathrm{n} / \mathrm{a}$ \\
\hline 1982 & 187 & 194 & $\mathrm{n} / \mathrm{a}$ & 1.78 & 4.85 & $\mathrm{n} / \mathrm{a}$ \\
\hline 1983 & 201 & 188 & $\mathrm{n} / \mathrm{a}$ & 4.0 & 43.70 & $\mathrm{n} / \mathrm{a}$ \\
\hline 1984 & 199 & 198 & $\mathrm{n} / \mathrm{a}$ & 40.70 & 43.47 & $\mathrm{n} / \mathrm{a}$ \\
\hline
\end{tabular}




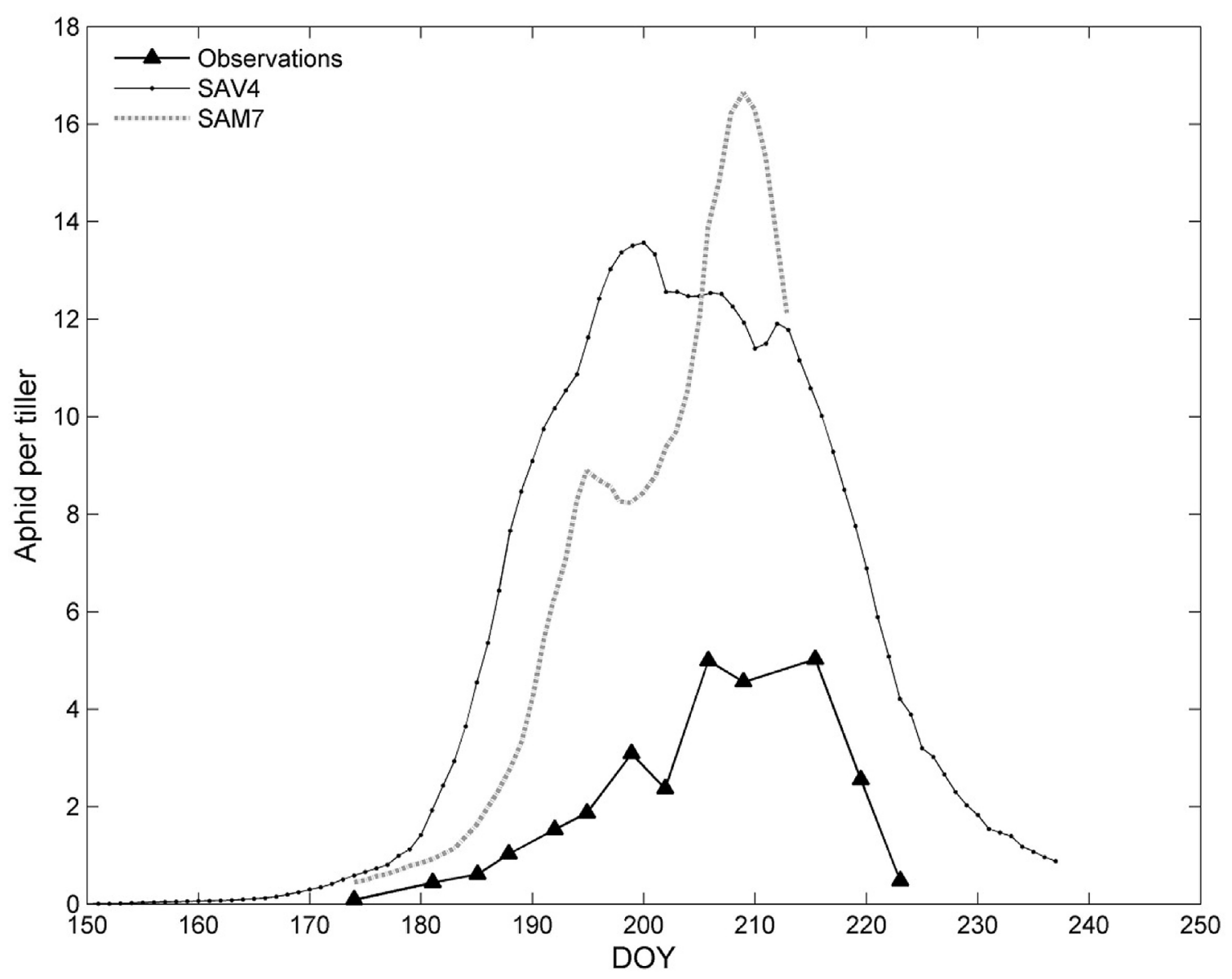

Fig. 10. Comparison of in-field aphid observations from 1978 in Norwich (cv:MH) with output from SAV4 and SAM7.

facilitate a direct comparison between each of the model's abilities to reproduce the peak observations. Overall, SAV4 performed well, particularly regarding the simulation of peak day occurrence (Fig. 11a). 1980 and 1981 are notable, in that the magnitudes of the observed and simulated peak days are significantly different. The discrepancy regarding 1980 can be explained owing to a malfunction in the suction trap at Brooms barn, resulting in intermittent catches between the 14th of May and the 1st of June that year. As a result, the aphid catch-data inputs to SAV4 were incomplete, which modified the input to the model. In the case of 1981, heavy rainfall has been implicated in retarding the population growth of $S$. avenae, by physically displacing the aphids from their host plant (Entwistle and Dixon, 1986). Overall however, SAV4 provided reasonable output when compared with the observations (Fig. 11).

\subsection{Sensitivity analysis}

The success of SAV4's evaluation provides a level of confidence in the model's ability to perform at least as well as previous models, if not better. However, it is important to recognise that model's such as the one described here, are always subject to uncertainty regarding the values of the numerous factors involved e.g. the developmental times, or the rate of survival. For this reason, Sensitivity Analyses are often employed, as they can serve to highlight (i) parameters which require additional research in the future in order to reduce output uncertainty, (ii) parameters or variables that 'add' nothing to the model and can essentially be removed and (iii) which parameter-driven inputs contribute the most to model variability (Hamby, 1994). The process is particularly useful, in that it serves to describe the general importance of a parameter, and by proxy the effort which should be invested in obtaining data to reduce uncertainty in that parameter.

Generally speaking, the method utilised for sensitivity analysis is determined by the computational practicality/ease of obtaining outputs. Due to the intensive computational requirements of SAV4, the run-time limits the extent to which a SA can be applied. For this reason, a small number of parameters deemed the most biologically significant to the dynamics of SAV4 were chosen for analysis. This method of SA is referred to as 'Screening', and is employed specifically in instances where model complexity is high and the number of parameters intractable. While this technique discriminates between parameters to be included in the SA, it provides some indication of the uncertainties associated with (what are deemed to be) the most influential parameters in the model. A 'local approach' (Cariboni et al., 2007) was employed, wherein the influence of the chosen parameters are tested by adjusting their values and maintaining all other variables as static. The parameters or inputs included in the SA were:

i. Nonlinear Lactin parameters used in the quantification of temperature-driven development.

ii. Temperature

iii. Survivorship parameter

In order to analyse the sensitivity of the model to a single parameter change at a time, a year of observed catches and temperatures, derived from the Rothamsted insect survey (Harrington and Woiwod, 2007), were utilised as input to SAV4. This was carried out due to the difficulty in disentangling potential sensitivities in the output if both a parameter of interest and the input aphid numbers 

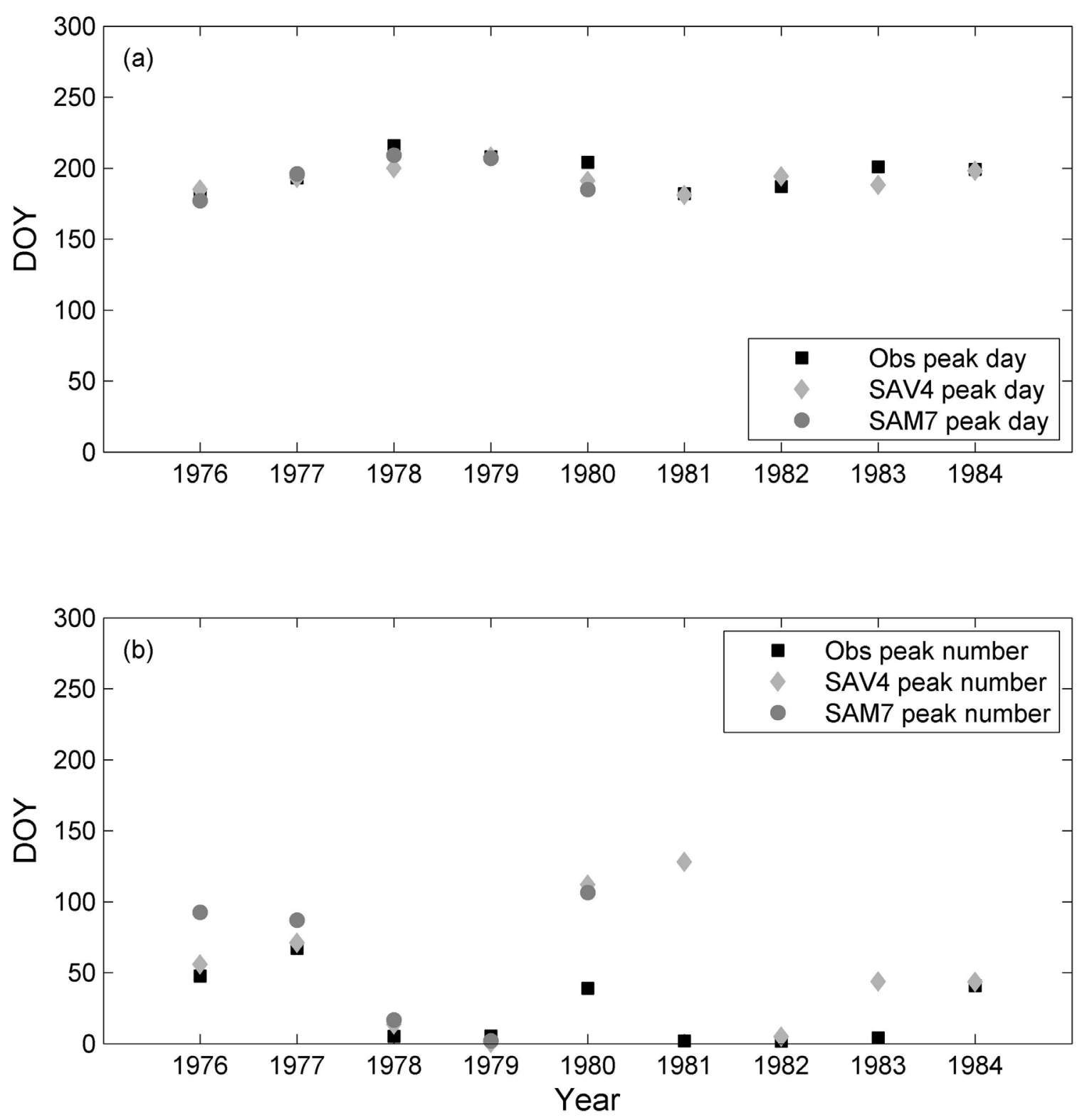

Fig. 11. Modelled and observed (Obs) (a) peak day (measured in DOY) and (b) peak number of aphids annually from 1976 to 1984 in Norwich.

are altered simultaneously. Fixed input data (temperature, catches, start and end dates) were utilised for each section of the SA below, ensuring that any resultant changes to the model output, were a result of the modified parameters, as opposed to changing input values.

\subsubsection{Lactin parameters}

The \pm standard errors around the mean developmental times for S. avenae were used in the SA to test the sensitivity of SAV4 to potential error in the Lactin parameters. The reported developmental time errors (Table 4) were added to the mean developmental time reported by Dean (1974) and these new values were used to refit the Lactin function in order to assess how the newly derived parameters (representing the \pm error around the developmental mean) would impact model output. These are illustrated in Fig. 12 for each of the instars in S. avenae.

The newly derived parameters, representing the \pm standard errors, were then separately employed in SAV4 in order to test their influence on the final model output. SAV4 outputs produced from three sets of Lactin parameters derived using: (i) the mean devel- opmental time, (ii) the mean developmental time minus the error and (iii) the mean developmental time plus the error are illustrated in Fig. 13. The output illustrated in Fig. 13 is consistent, in that the 'plus error' output produces slightly lower peak numbers, due to the lengthening of the developmental period, ultimately elongating the time to adulthood and reproduction, and lowering population numbers. In the case of the 'minus error', the opposite is the case. The changes in the timing of the peak numbers and the peak numbers themselves in response to the SA are small, suggesting that SAV4 is not overly sensitive to changes in the parameters (assuming that their values are derived from data that lies within the spread recorded in the initial lab studies (Dean, 1974)).

\subsubsection{Temperature}

Two temperature increments were chosen by which to perturb the model: (i) $\pm 2{ }^{\circ} \mathrm{C}$ and (ii) $\pm 4{ }^{\circ} \mathrm{C}$. These increments were considered of ample magnitude to provide a range over which SAV4's sensitivity could be assessed. The SA suggested that SAV4 is particularly sensitive to temperature, indicating large differences between outputs when temperature was modified by $\pm 2 / 4^{\circ} \mathrm{C}$. 

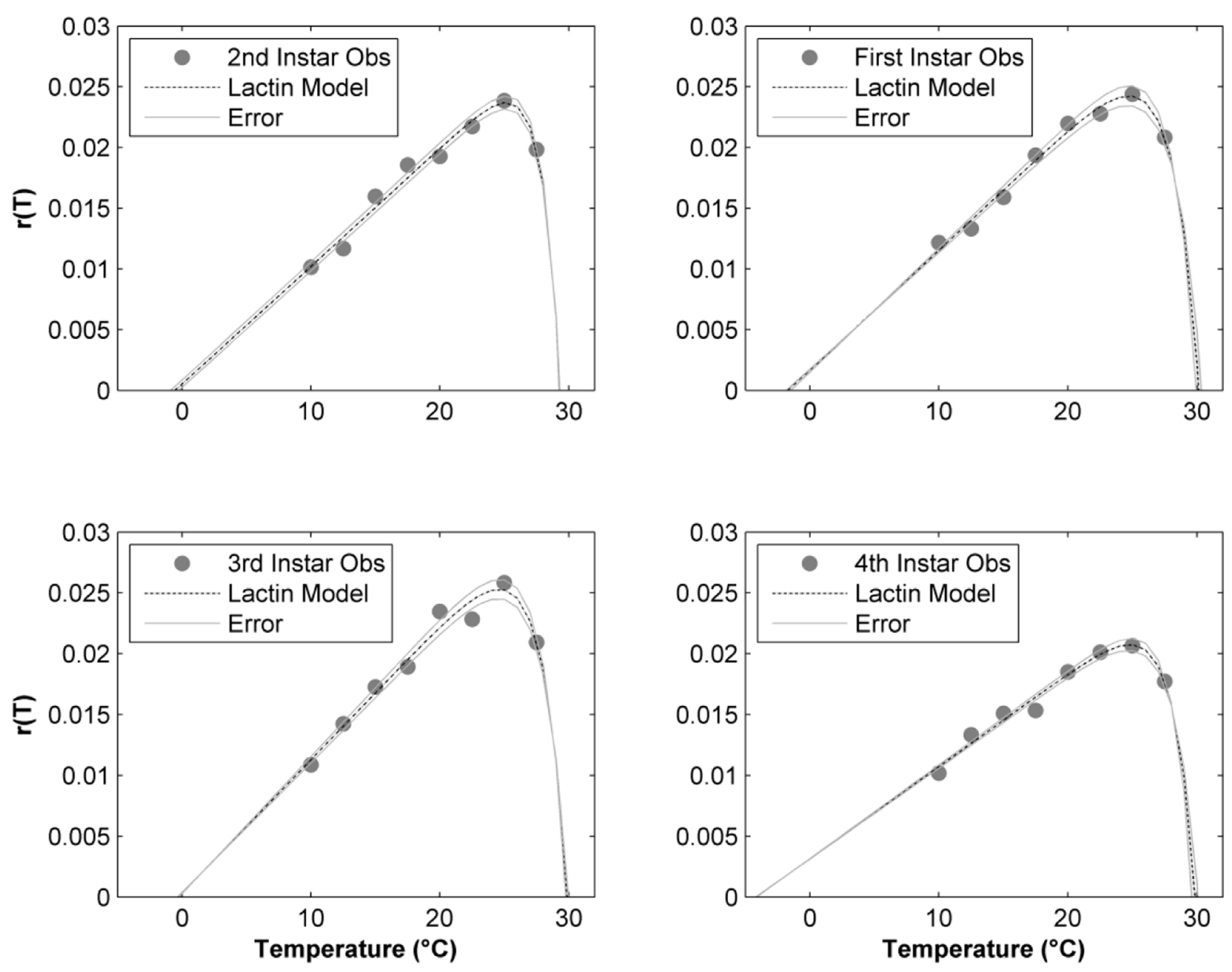

Fig. 12. Original Lactin model fit (black dashed line) and \pm Lactin model fit based on the incorporation of the standard error (grey lines).

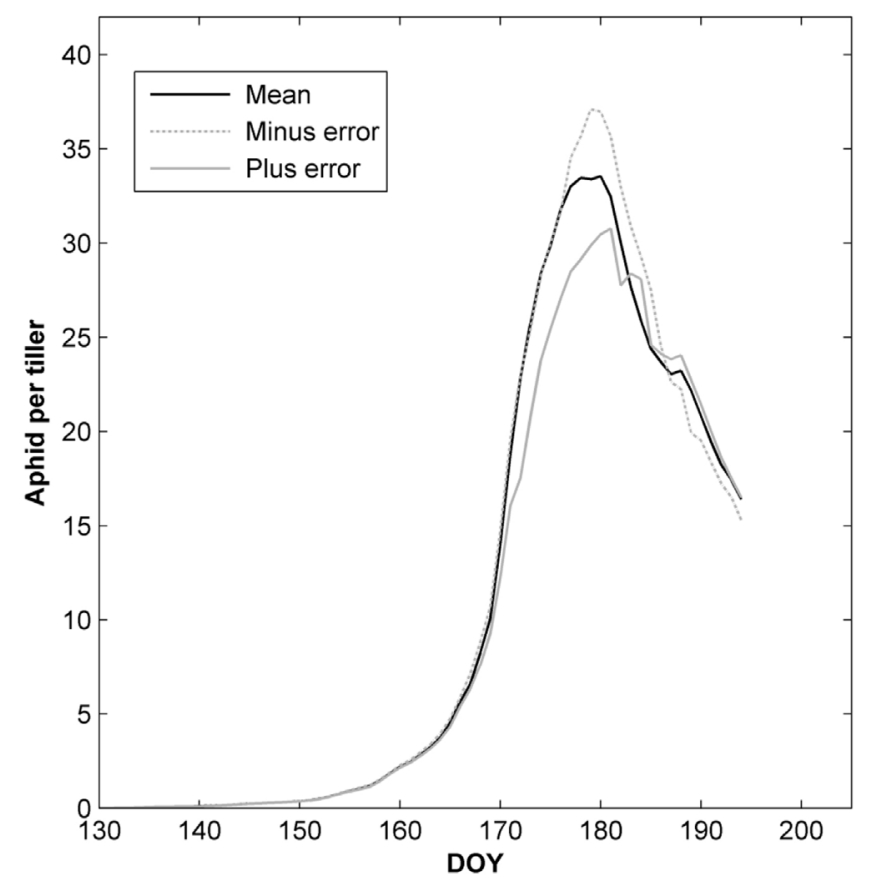

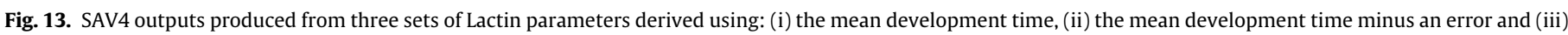
the mean development time plus an error. 

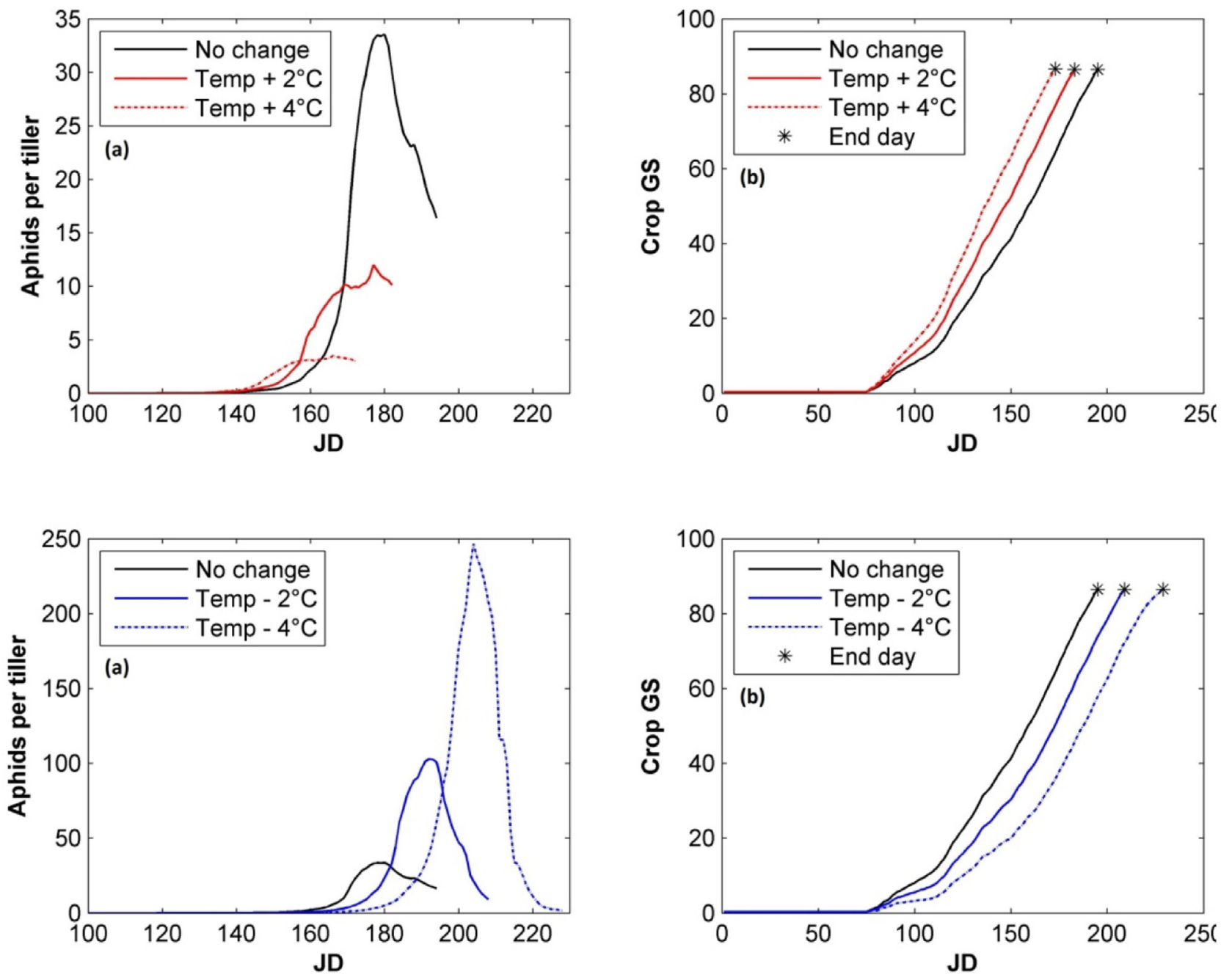

Fig. 14. SAV4 output from moderate-regime temperature SA. Magnitude of output response to (a) increased temperatures and (b) decreased temperatures.

This finding is not surprising, considering the dependence of the model-dynamics on temperature. The relationship between final model output and temperature increase is revealed as a linear one, although not in the direction that one might expect. Increases in temperature across all of the regimes produced a consistent decrease in APT output, while decreases in temperature precipitated APT increases. This is perhaps counterintuitive to what would have been expected considering the relationship between temperature and insect development, however the mechanisms which drive this negative linear relationship can be explained.

There appears to be two processes driving the sensitivity illustrated in Fig. 14. Increases in temperature facilitate an earlier and more pronounced population-increase in the 'increasedtemperature' model population, due to the earlier onset of sexual maturity as a result of the increased rate of temperature-dependent development (particularly evident in Fig. 14(a)). This increase in density over a short period of time promotes the production of progressively higher numbers of alates owing to crowding, resulting in population decline. Simultaneously, the increased thermal energy in the system also serves to advance the timing of the critical crop GS's, capping the growth of the population (due to the earlier occurrence of GS's which were unsuitable for aphid hosts). Ultimately, SAV4 appears to be highly sensitive to temperature, due to the phenological relationship between the model population and their host plant. This sensitivity is not viewed however, as a negative aspect of the model. To the contrary, the SA serves to bolster confidence in the model, as large changes in the most important driver (temperature) promoted systematic and logical changes in SAV4 output. It is worth noting however, that while this section of the SA highlights the sensitivity of SAV4 to temperature inputs, it does not necessarily indicate the expected directionality of the final model output in response to increasing temperatures, due to the unrealistic nature of the 'static' model inputs for the purpose of the SA. This is an area that merits further analysis however, to test how the model outputs may change in response to modelled future temperature inputs as a result of climate change.

\subsubsection{Survivorship}

Survival percentage was altered by $\pm 5 \%$ for each of the temperature regimes, resulting in an unequivocal linear increase in output when survival was increased, and a decrease in output when survival was decreased (Fig. 15). No effects of interactive processes were evident in the output. The magnitude of the divergence in outputs across each of the survival levels, suggests that SAV4 is sensitive to this input parameter. The strong linear relationship evident in SAV4 output when the survival rates are altered, highlights the importance of the accuracy of the survival submodel. Since a simplistic rendering of survival was implemented in SAV4 (as described in Section 2.7), this SA has highlighted an area which merits more effort in data acquisition if uncertainty derived from this input is to be reduced in future applications. 


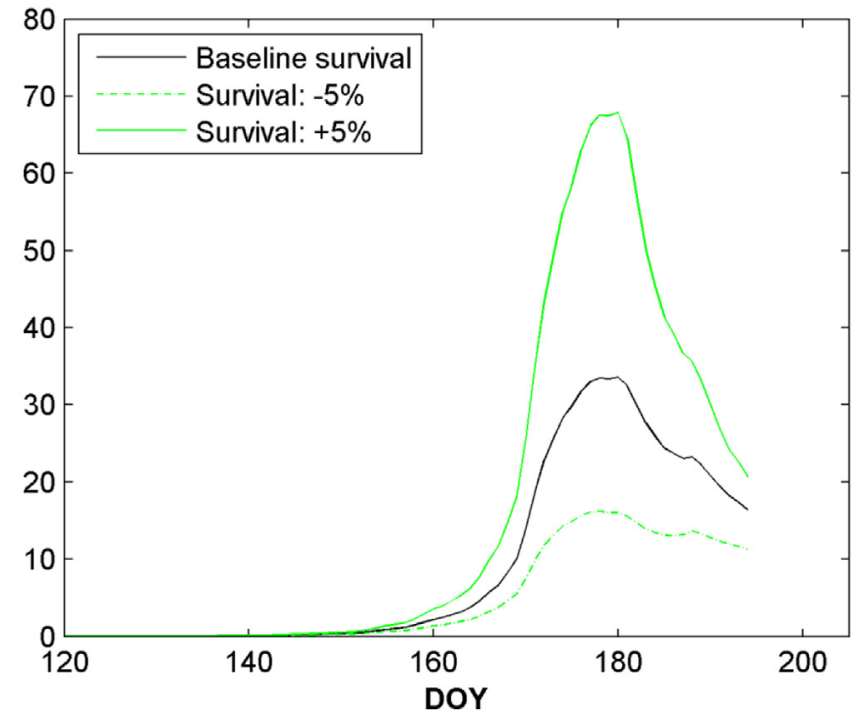

Fig. 15. SAV4 output using two different levels of survivorship.

\subsection{Discussion}

The model presented here provides a framework for assessing seasonal S. avenae population progression in response to daily temperature. This model is not the first simulation model to describe the population dynamics of the grain aphid, however it has been shown to constitute an improvement to previous simulation models. The incorporation of the Lactin model to describe the nonlinear developmental response in $S$. avenae to temperature is a step forward in relation to simulating this species dynamics. Specifically, the incorporation of biologically interpretable parameters, coupled with this function's ability to provide meaningful estimates of all the pertinent developmental thresholds is a major advantage when describing species dynamics. In particular, the former is a major model asset, as it not only describes, but also endeavours to explain the relationship between development and temperature in terms of the underlying physiological mechanisms (Walgama and Zalucki, 2006). The incorporation of this type of approach improves upon the most recent work of Skirvin et al. (1997), whose attempt to improve on SAM7 by incorporating a measure of nonlinearity, did not capitalise fully on optimising the development submodel to incorporate biological realism. As a result, even in the absence of the further model modifications introduced here, the incorporation of the Lactin model serves to provide a level of biological meaningfulness not previously incorporated in either SAM7 or SACSIM. The evaluation of SAV4 and its direct comparison with previous models supports the assertion that SAV4 constitutes an improved tool to model $S$. avenae in response to temperature.

SAV4 is not without its limitations; and 1981 serves to highlight an issue with the model's capacity to describe aphid dynamics under extreme conditions. As referred to above, the occurrence of particularly heavy rainfall during the evaluation period for 1981 could have been responsible for the discrepancy that exists between model output and observations. This is an area that merits further investigation, to assess if it would be beneficial to incorporate some aspect of rainfall into SAV4. The activity of natural enemies could also be responsible for the discrepancies between observed and modelled peak numbers, however in the majority of evaluation years addressed above, the exclusion of natural enemies from the model does not appear to have impacted SAV4's ability to provide outputs comparable to the observations. Indeed, the metrics calculated by SAV4 were in all cases at least as good, if not better than the outputs derived from previous models. Skirvin's model outputs directly incorporated natural enemy subroutines, while SAM7 outputs incorporated reduced predation. Despite this consideration of natural enemies, both SAM7 and SACSIM produced peak numbers in excess of SAV4's outputs. This finding suggests that it is possible to capture the dominant signal in the population dynamics of the grain aphid without the requirement for a data-hungry predator submodel. That is not to suggest that the exclusion of natural enemies will always provide the 'best' or most skilful model, but rather that in the absence of data describing the entire guild of natural enemy activities, results garnered using a one or two natural enemies (as in SAM7 and SACSIM) may not improve the model outputs enough to justify the data-intensive requirements. This is an area that merits further work, in order to disentangle or quantify the effects of these limiting factors on aphid populations. Indeed, it is an area that could be assessed for future addition to the model presented here, in an effort to incorporate additional facets of the aphid's lifecycle.

Despite the limitations highlighted here, SAV4's outputs have been shown to reflect real aphid population dynamics, in particular the timing of aphid population peaks. This type of functionality has the potential to be invaluable within a Decision Support System (DSS) as an early warning system, or as a long-term climate change scoping tool. It is expected that models such as SAV4 will become more implicit in DSS in the future, particularly in light of legislation such as the European Sustainable Use of pesticides Directive (SUD), which explicitly mandates the consideration of knowledge-based decision-making regarding the application of chemical controls.

The limited SA carried out on SAV4 provided reassuring evidence that the model responds in a logical and systematic manner to changes in (what were considered to be) some of the most influential parameters. This part of the analysis also served to highlight some areas that merit future work, if SAV4 is to be improved in the future. Firstly, the counterintuitive manner in which the model outputs shifted in response to increasing and decreasing the temperature inputs (decreased aphid numbers and increased aphid numbers respectively), highlights an area which merits further attention: the use of SAV4 as a predictive tool for climate change studies. It also provides insight and a more fundamental understanding of the underlying driving processes, the essence of any modelling endeavour. Future work could incorporate the use of modelled temperature inputs to test if aphid populations respond positively or negatively to increasing environmental temperatures, when all other facets of the model are allowed to respond dynamically to the data inputs. For example, increases in temperature inputs would likely affect the timing of the immigration start and end, as well as its composition (data which remained static for the SA here). The model was also found to be sensitive to the survival algorithm, highlighting this area as one which demands further research and data collection efforts, in order to improve upon the pragmatic approach used in SAV4.

\subsection{Conclusions}

The model presented here constitutes an improved system to simulate the temperature-dependent population dynamics of $S$. avenae in cereal crops. Ultimately, the contribution that SAV4 constitutes should be interpreted and contextualised within an Integrated Pest Management paradigm. The ultimate aim of models within the context of pest science is twofold: (1) to optimise the timing, location and nature of control strategies and (2) to facilitate investigations regarding future population responses to environmental stimuli (e.g. climatic change). The current work addresses the former aim by providing a new and improved tool for modelling aphid pest dynamics in response to temperature. The model has been shown to provide acceptable outputs when assessed within a multi-year evaluation period, and as a result mer- 
its further assessment regarding its potential utility as a DSS tool. The latter aim highlights the potential for further development of the model, wherein modifications could facilitate the ingestion of climate change scenarios, to explore potential alterations to aphid population dynamics in a future warmer climate.

\section{Role of funding source}

This research was funded by Teagasc, the agricultural and food development authority in the Republic of Ireland under the Walsh Fellowship scheme.

It was the decision of the authors to submit this manuscript for publication.

\section{Appendix A. SAV4}

\section{A-1 Loading all the temperature data}

a11_tmax_yrs=1oad ('maximum_temperature.csv');

a11_tmin_yrs=1oad( 'minimum_temperature.csv');

mod_temp_counter $=0$;

A-2 Time and sunrise calculation

for mod_temp $=1:\left(\operatorname{size}\left(a 11 \_t m a x \_y r s, 2\right)\right)$;

mod_temp_counter $=$ mod_temp_counter +1 ;

disp(['year number ' num2str(mod_temp_counter)]);

\%Save the 'year' of the temperatures

sim_year $=[1976: 1984]^{\prime}$;

mode1_year(mod_temp_counter) = sim_year(mod_temp_counter);

\%The next lines provide the date for the calculation of sunrise

date $=$ load('yrmthdy.csv');

year $=\operatorname{date}(:, 1)$;

month $=\operatorname{date}(:, 2)$;

calday $=\operatorname{date}(:, 3)$;

\%Enable next 2 lines when multiple locations are being used

$\% 1$ at = input ('Please insert latitude an as integer: ')

\%1on = input('Please insert longitude an as integer: ')

\%sunrise = suncycle(1at, 1on, [year, month, calday],2880) *sunrise function;

sunrise $=$ suncycle $(57,-6$, [year, month, calday $], 2880)$;

$\mathrm{T}=\left[\left(\mathrm{a} 11\right.\right.$ _tmax_yrs $\left.\left(:, \bmod \_t e m p\right)\right),\left(\right.$ a $\left.\left.11 \_t m i n \_y r s\left(:, \bmod \_t e m p\right)\right)\right]$;

\%This calls up the sinewave function to produce hrly temps for the \%mode1.

\% You need to specify the dlytemps in ' $T$ ' (*sinewave function);

Temp $\quad$ sinewave $(T$, sunrise); 


\section{A-3 Initialising variables}

\begin{tabular}{|c|c|}
\hline a1_nymph_percentage & $=0$; \\
\hline a1_repro_rate & $=0$; \\
\hline alighting_ad & $=0$; \\
\hline alighting_yearly & $=0$ \\
\hline ap_nymph_percentage & $=0$; \\
\hline ap_repro_rate & $=0$; \\
\hline aphid_per_ti11er & $=0$; \\
\hline currentday & $=0$; \\
\hline dai7yage & $=0$ \\
\hline day & $=0$; \\
\hline daycounter & $=0$ \\
\hline daystart & $=0$; \\
\hline DD & $=0$ \\
\hline end_of_migrat & $=0$; \\
\hline endday & $=0$ \\
\hline end_4th_day & $=0$ \\
\hline end_4th_hr & $=0$; \\
\hline fins_duration & $=0$; \\
\hline $\begin{array}{l}\text { tiller } \\
\text { clear g; }\end{array}$ & $=0$; \\
\hline GS & $=0$; \\
\hline gs_jd & $=0$; \\
\hline gsday & $=0$ \\
\hline $\mathrm{Hh}$ & $=0$; \\
\hline $\mathrm{hr}=1$ & \\
\hline jd32_200_av & $=0$; \\
\hline jf_av & $=0$; \\
\hline myfileId & $=0$ \\
\hline maxday & $=0$; \\
\hline num_a1_nymphs & $=0$; \\
\hline num_ap_nymphs & $=0$; \\
\hline numdays & $=0$; \\
\hline nymphs_a1_parent & $=0$; \\
\hline nymphs_ap_parent & $=0$; \\
\hline peak & $=0$; \\
\hline regime & $=0$; \\
\hline stage & $=1$ \\
\hline clear surviva1_percen & \\
\hline survivalI & $=0$ \\
\hline system_a1_day & $=0$; \\
\hline tot_d7y_nymph & $=0$; \\
\hline tota1_number & $=0$; \\
\hline $\begin{array}{l}\text { \%Temp data for th } \\
\text { dlytmax } \\
\text { d7ytmin } \\
\text { d7ytav }\end{array}$ & $\begin{array}{l}\text { reproduction \& CDD_new functions } \\
=\mathrm{T}(:, 1) \\
=\mathrm{T}(:, 2) \\
=(\text { d } 7 \text { ytmin+d7ytmax }) / 2\end{array}$ \\
\hline $\begin{array}{l}\% \text { Simulate the sta } \\
\text { daystarts = load } \\
\text { daystart }=\text { dayst } \\
\text { daily_nympha1_sur }\end{array}$ & $\begin{array}{l}\text { tday based on the previous mean Jan/Feb temperature } \\
\text { start.csv'); } \\
\text { rts(mod_temp,1); } \\
\text { iva1 = zeros(daystart, endday); }\end{array}$ \\
\hline
\end{tabular}


\%This variable is for storing each years startday

store_startday (mod_temp) = daystart;

\%Load the endday of migration

migration_ending = load ('end.csv')

end_of_migrat = migration_ending(mod_temp,1);

\%This variable is for storing each year's end of migration jd

store_end_migrat(mod_temp) = end_of_migrat;

sow_date $=76$;

\%This calls up the cumulated degree day function, (*CDD_new function)

DD = CDD_new(dlytav, sow_date);

\%This calls up the crop growth stage function, (*ZGS function)

\%GS is the crop growth stage in Zadoks decimal scale

GS $\quad$ ZGS(DD);

gsday $=(1: \text { length (GS) })^{\prime}$;

\%End the model run when the GS becomes unsuitable

gs_jd = horzcat(GS, gsday);

for $\mathrm{g}=1:$ length(GS);

if $\mathrm{GS}(\mathrm{g})>=86.3$;

endday $=$ gs_jd $(\mathrm{g}, 2)$;

store_endday (mod_temp_counter,1) = endday;

break

end

end

cum_a1n = zeros (endday, 1$)$;

cum_apn $\quad$ zeros (endday, 1$)$;

\%This calls up the alate reproduction rates (*al_repro_func function)

a1_repro_rate $\quad$ a1_repro_func $(\mathrm{dl} y \mathrm{tav}, \mathrm{GS})$;

\%This calls up the apterous reproduction rates (*ap_repro_func function)

ap_repro_rate = ap_repro_func $($ dlytav, GS $)$;

numhrs $\quad=\operatorname{length}(\operatorname{Temp}(:, 1))$;

numdays $\quad=$ endday-daystart;

dev $\quad=$ zeros (numhrs, endday, endday);

age $\quad=$ zeros (numhrs, endday, endday);

stage $\quad=1$;

reproducing_ap = zeros (endday, 1 );

reproducing_al = zeros (endday, 1$)$;

system_ap_day = zeros(endday, 1 ) :

\%Initialise variables required for recording the time of maturation

alighting_daystart_endday $=0$;

caught $=0$;

devdaystart $\quad=0$;

devdayend_5th $\quad=0$;

devhrend_5th $\quad=0$;

dev_duration_days_5th $\quad=0$;

dev_duration_days_hrs_5th $=0$;

endof5th_instar $\quad=0$;

dev_duration_hrs_5th $\quad=0$;

dev_duration_5th_rounded_days $=0$;

devdayend_4th $\quad=0$;

devhrend_4th $\quad=0$; 


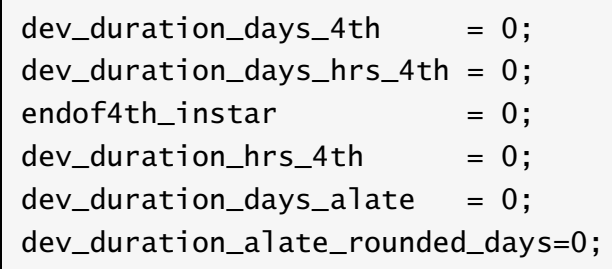

A-4 Seeding the model with simulated aphid numbers

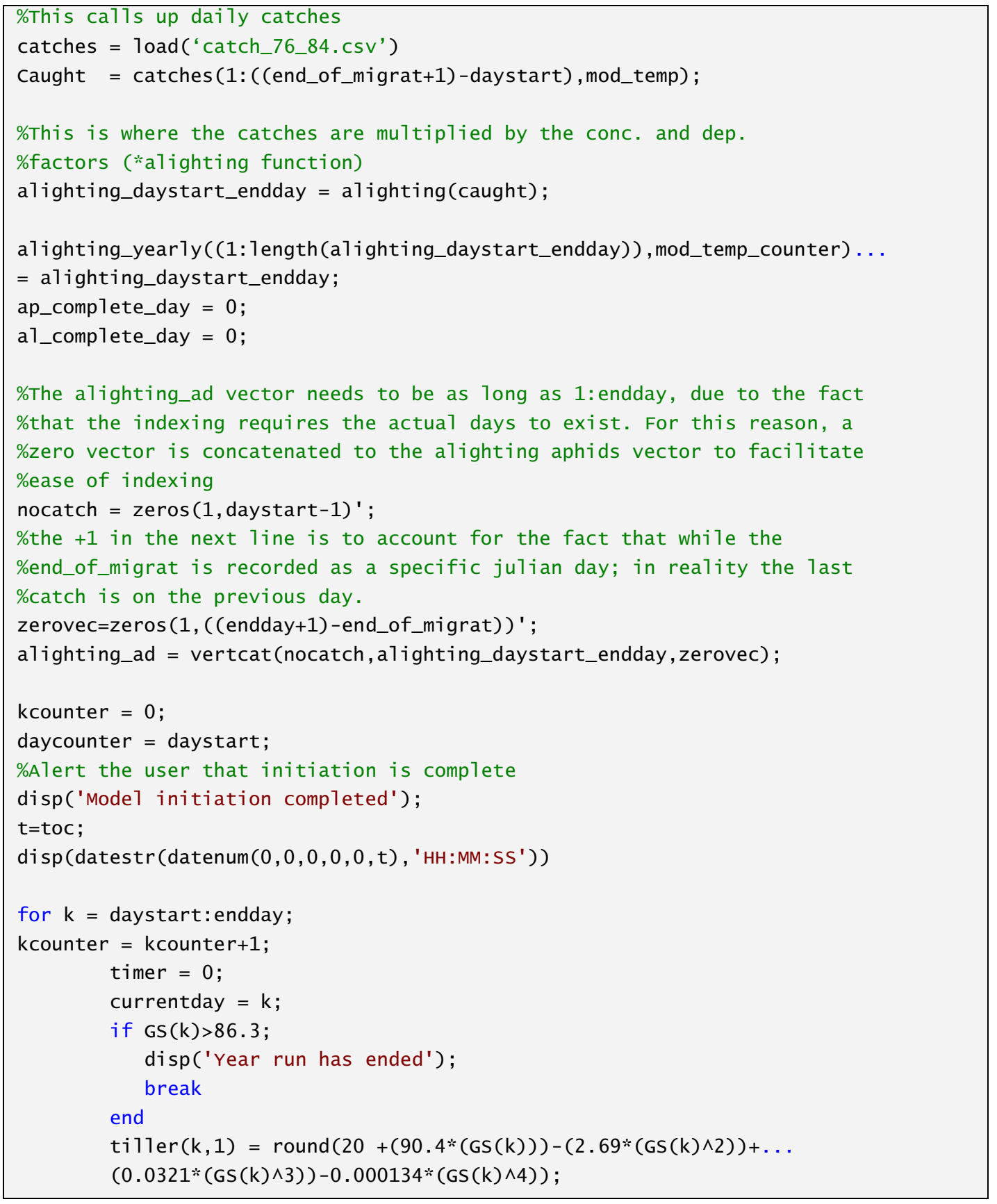


A-5 Calculate the number of reproducing individuals

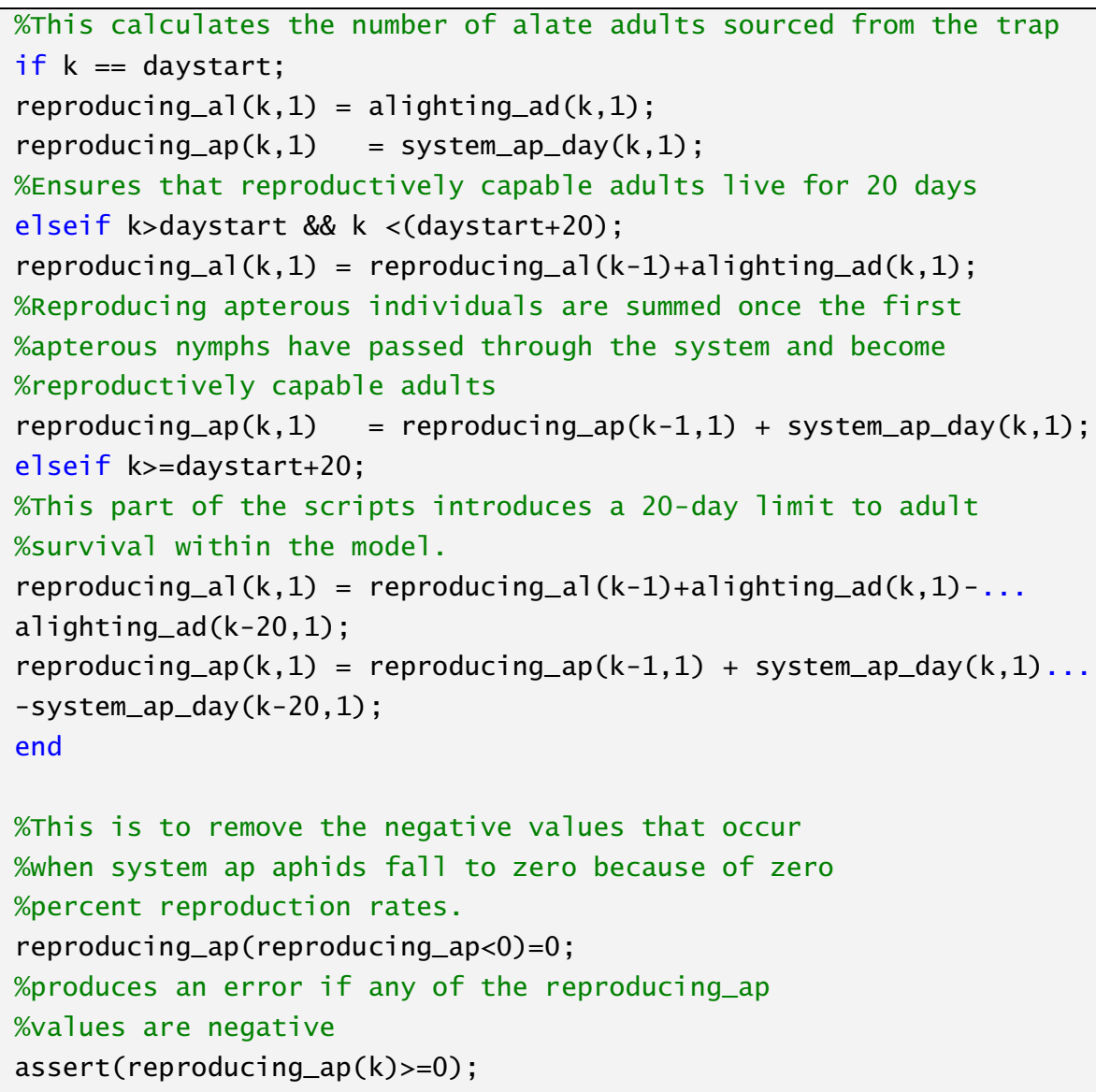


A-6 Calculate the daily nymphs produced

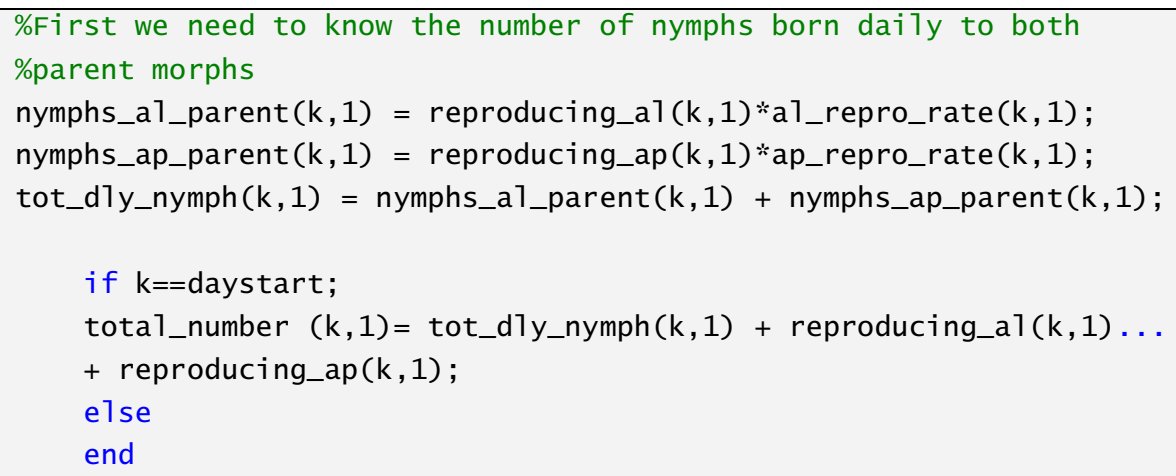


A-7 Development submodel

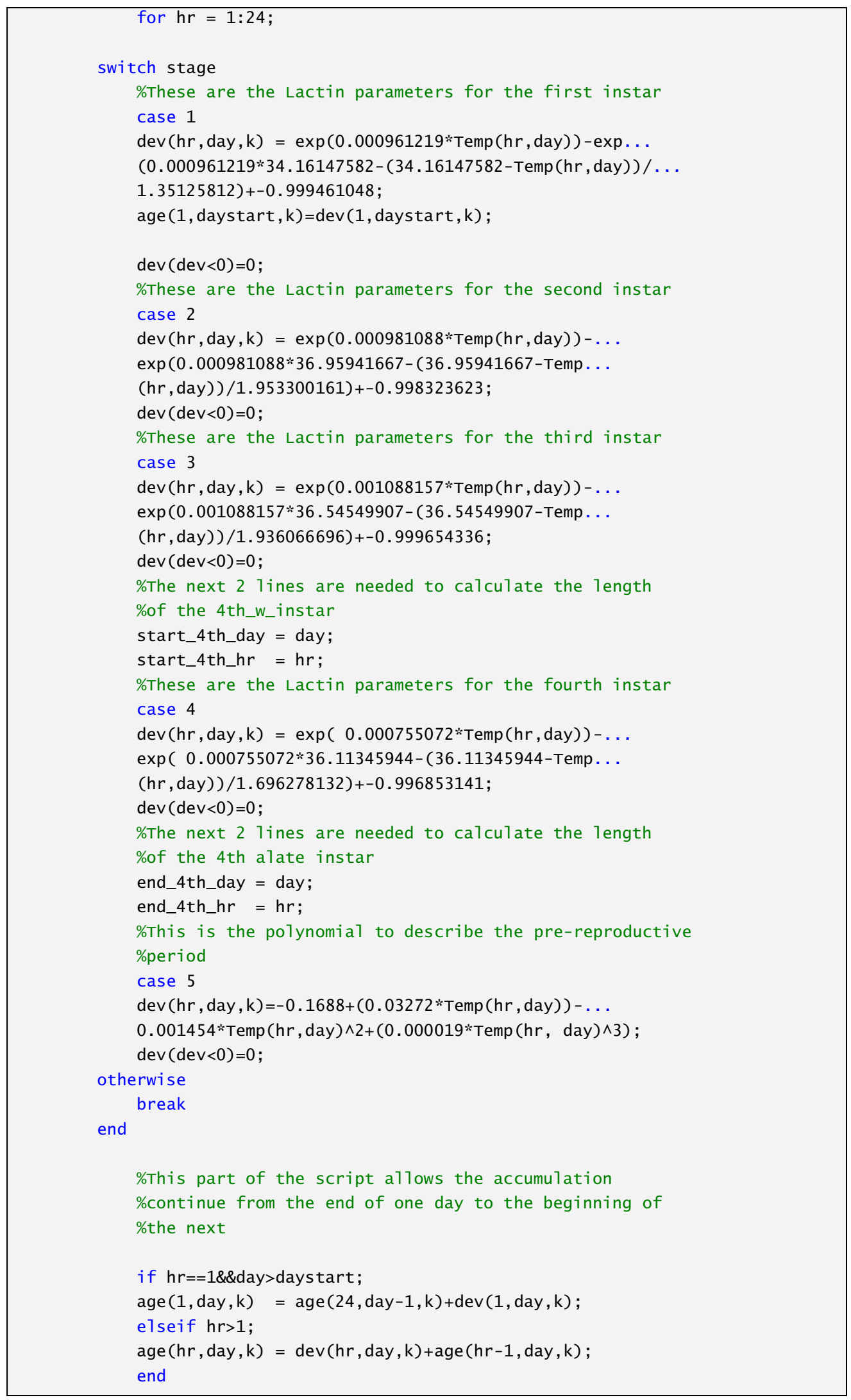


\%This selects the developmental stage parameters to use

if $\operatorname{age}(\mathrm{hr}$, day, $\mathrm{k})<=1$;

stage $=1$;

elseif age $(\mathrm{hr}$, day, $\mathrm{k})>1 \& \&$ age $(\mathrm{hr}$, day, $\mathrm{k})<=2$;

stage $=2$;

elseif age $(h r$, day, $k)>2 \& \&$ age $(h r$, day,$k)<=3$;

stage $=3$;

elseif age $(\mathrm{hr}$, day, $\mathrm{k})>3 \& \&$ age $(\mathrm{hr}$, day, $\mathrm{k})<=4$;

stage $=4$;

\%stop accumulating once age reaches approximately 4

e1seif age $(\mathrm{hr}$, day, $\mathrm{k})>4$ \&\&age $(\mathrm{hr}$, day, $\mathrm{k})<=5$;

stage $=5$;

e1se

\%The day development ends

$\operatorname{devdaystart}(\mathrm{k}, 1)=\mathrm{k}$;

devdayend_5th $(k, 1)=$ day;

$\%$ The $\mathrm{hr}$ development ends

devhrend_5th $(k, 1)=h r$;

\%Gives the number of days the nymphs developed for

dev_duration_days_5th = devdayend_5th-devdaystart;

$\%$ Gives the number of days and hours the nymphs

\%developed for

dev_duration_days_hrs_5th $=$ [dev_duration_days_5th...

devhrend_5th] ;

\%Provides a matrix with daystart(:,1), dayend(:,2) and

\%hrend (: , 3)

endof5th_instar $=$ [devdaystart devdayend_5th devhrend_5th] ;

$\%$ These lines are rounding the duration of 5 th development to $\%$ the nearest day

dev_duration_hrs_5th $=$ (dev_duration_days_5th*24)+devhrend_5th;

dev_duration_5th_rounded_days = round (dev_duration_hrs_5th/24);

ap_complete_day = devdaystart + dev_duration_5th_rounded_days;

break

end

\%When the age in any cel1 reaches 4 (i.e. end of 4 th instar

if age $(h r$, day, $k)>=4$ \&\& timer $==0$;

\%The number of new adults for that dimension ' $k$ ' is indexed by

\%matching $k$ (the initialising day for those nymphs) to ' $k$ ' of the

\%nymph vector

timer $=1$;

\%The day development begins

$\operatorname{devdaystart}(k, 1)=k$;

\%The day development ends

devdayend_4th $(k, 1)=$ day;

\%The $\mathrm{hr}$ development ends

devhrend_4th $(\mathrm{k}, 1)=\mathrm{hr}$;

\%Gives the number of days the nymphs developed for

dev_duration_days_4th=devdayend_4th-devdaystart;

\%Gives the number of days and hours the nymphs developed for

dev_duration_days_hrs_4th=[dev_duration_days_4th devhrend_4th] ;

\%Provides a matrix with daystart $(:, 1)$, dayend(:,2) and hrend(:,3)

endof4th_instar=[devdaystart devdayend_4th devhrend_4th]; 
\%Provides the number of hrs it took to reach the 4 th instar

dev_duration_hrs_4th=(dev_duration_days_4th*24)+devhrend_4th ;

\%Alates take $x 1.5$ times longer in the 4 th instar to mature than an \%apterous 4th instar

\%the next line provides half the time ap_4th takes in hours

fins_duration $(k, 1)=((($ end_4th_day*24) + end_4th_hr $)-\ldots$

((start_4th_day*24)+start_4th_hr))/2);

dev_duration_days_alate $=$ (dev_duration_hrs_4th+...

fins_duration(k))/24;

\%Provides the number of days it took to develop to an alate adult

dev_duration_alate_rounded_days=round(dev_duration_days_alate);

\%This provides the day on which the alates complete development and

\%leave the crop by adding the development duration to the day

\%they were born

a1_complete_day $(\mathrm{k}, 1)=\operatorname{devdaystart}(\mathrm{k})+\ldots$

dev_duration_alate_rounded_days $(k, 1)$;

else

end

end

A-8 Survival

dailyage $($ day,, $\mathrm{k})=$ age $(1$, day, $\mathrm{k})$;

\%The daily accrued development

Hh $($ day, $\mathrm{k})=$ dailyage $($ day, $\mathrm{k})$-dailyage $($ day-1, $\mathrm{k})$;

\%This part of the script produces daily nymphal

\%survival

if $\mathrm{GS}($ day) $<73$;

surviva1_percent $($ day, 1$)=94.4449-0.0000000332214 * \ldots$

$(\exp (0.725604 * \mathrm{~d} 7 \mathrm{ytav}($ day, 1$)))$;

e1se

surviva1_percent $($ day, 1$)=45$;

end

survivalI $($ day, 1$)=($ survival_percent $($ day, 1$)) / 100$;

$\mathrm{Hi}=1$;

dai1y_nympha1_survival $($ day, $k)=(\operatorname{survivalI}($ day, 1$) \wedge(H h($ day,$k) / H i))$;

\%Survival applied to daily nymphs

num_a1_nymphs $(k, 1)=$ num_a1_nymphs $(k, 1) * \ldots$

dai1y_nympha1_surviva1 (day,k);

num_ap_nymphs $(k, 1)=$ num_ap_nymphs $(k, 1) * \ldots$

dai7y_nympha1_survival (day,k); 


\section{A-9 Calculates the apterous cohort}

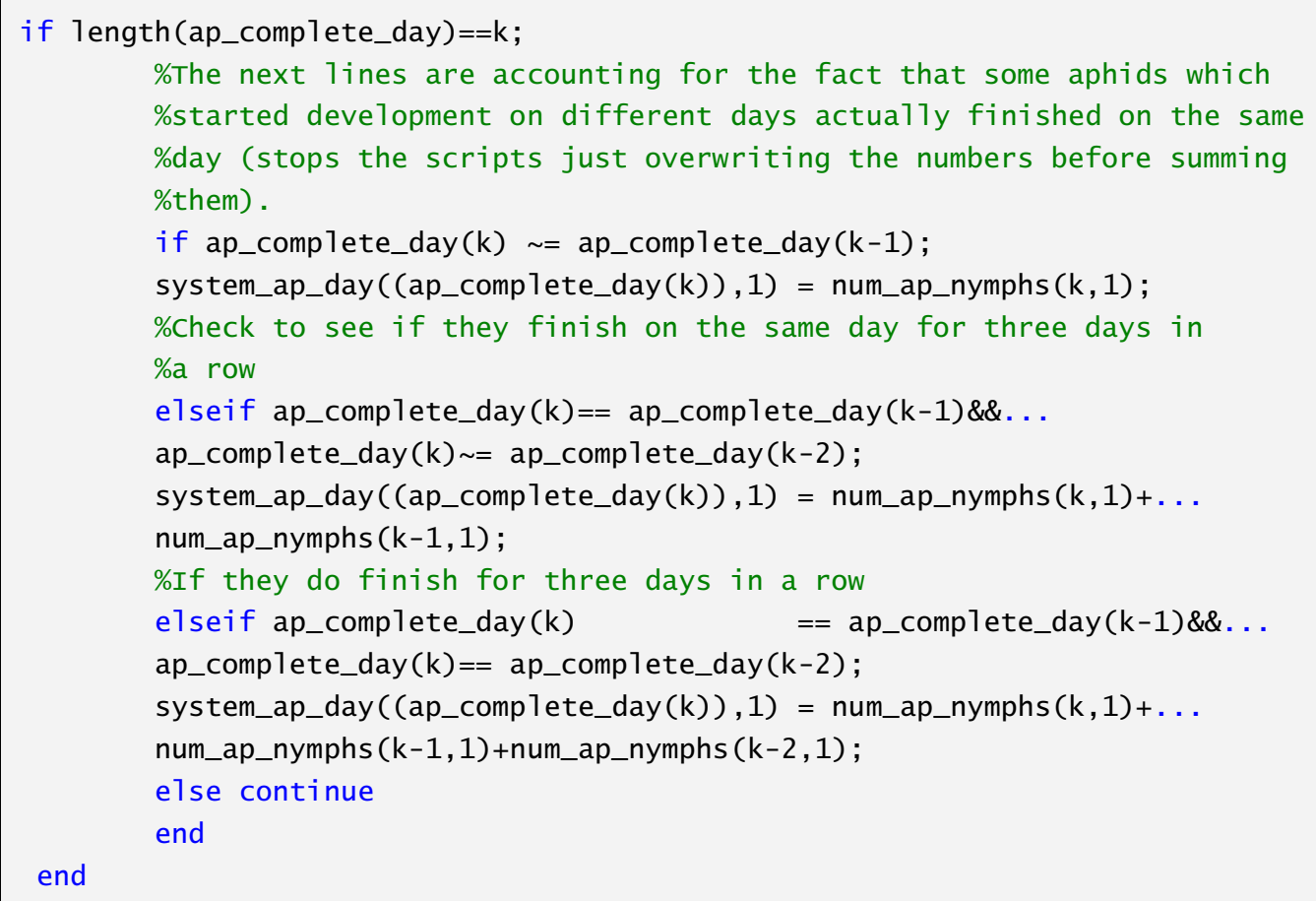

A-10 Calculates the alate cohort

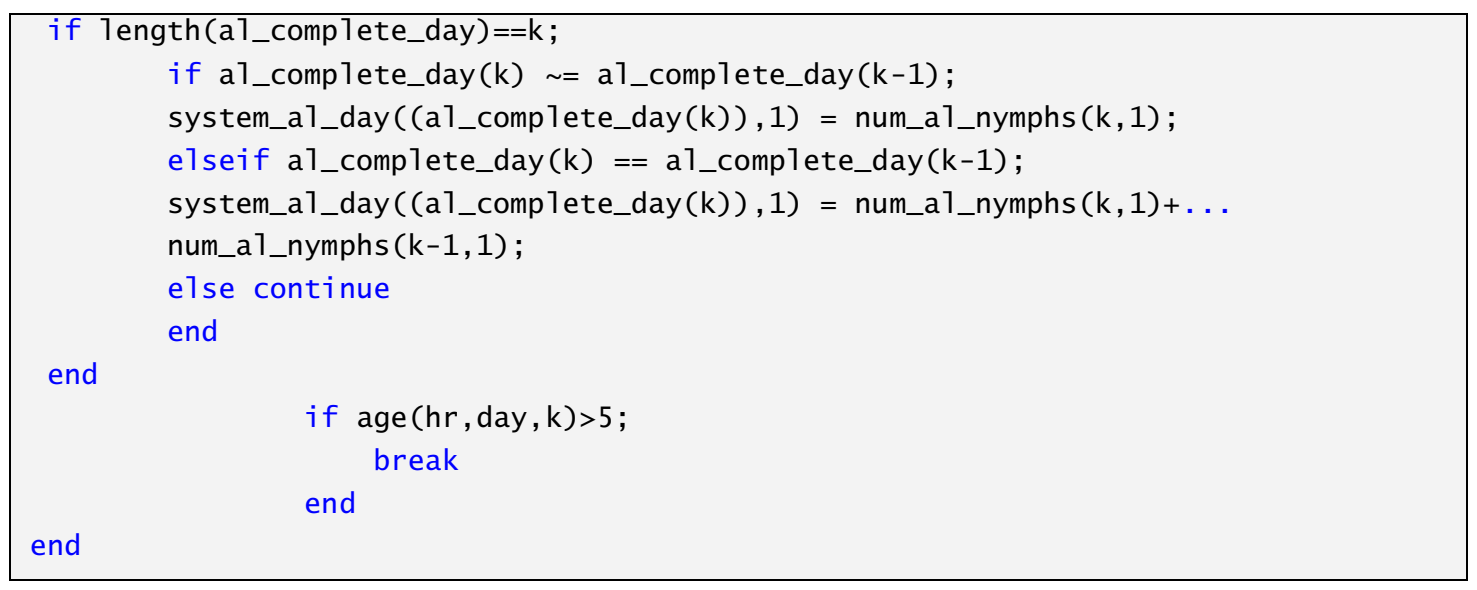




\section{A-11 Cumulative numbers}

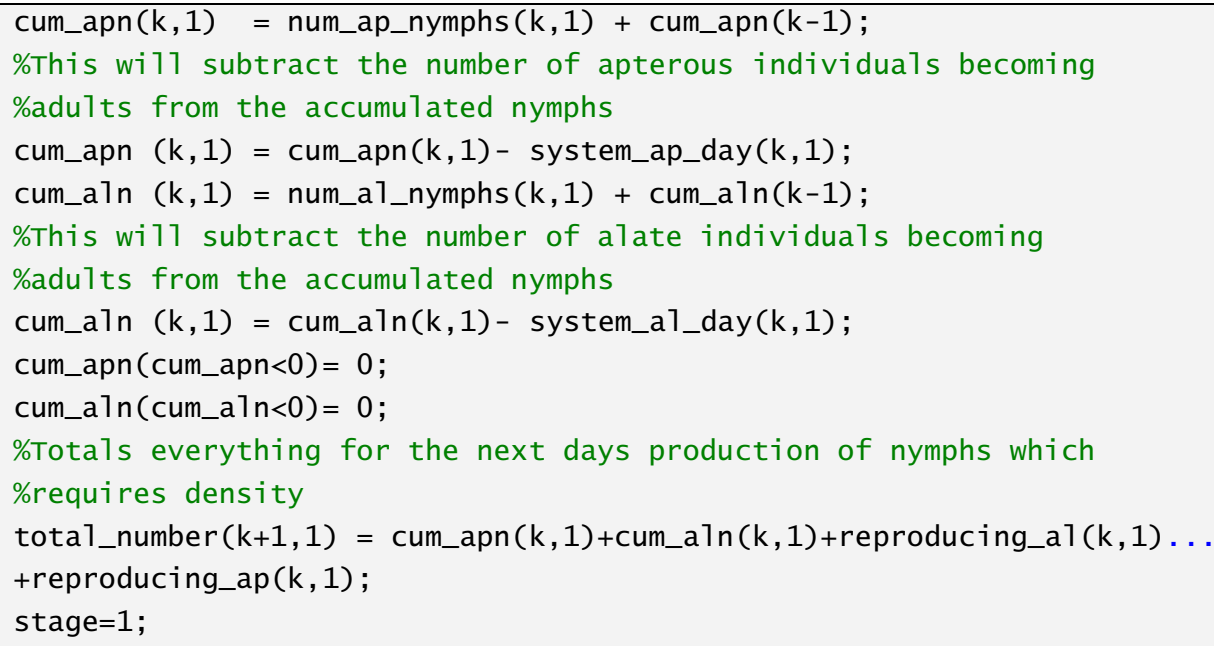

A-12 Store aphid metrics

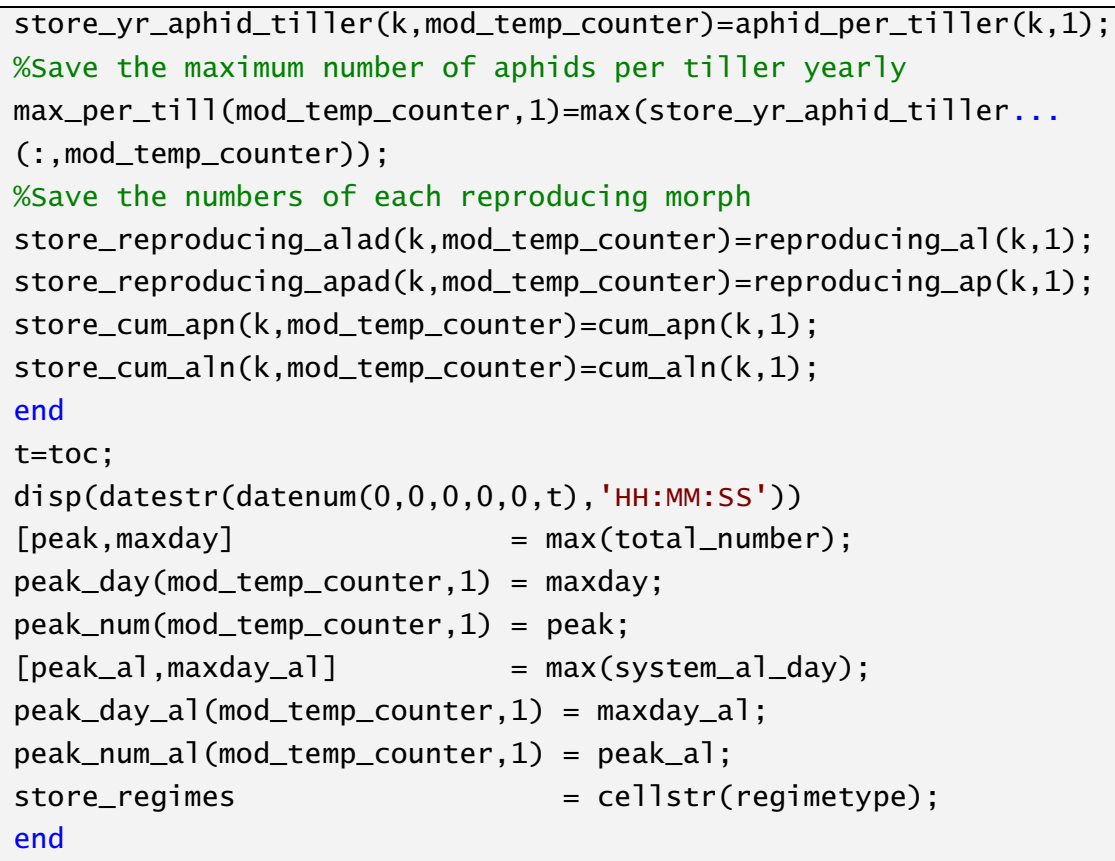

A-13 Save the model output and end

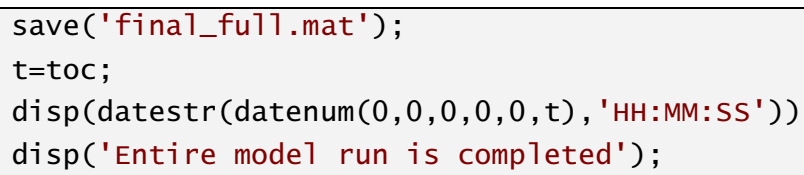




\section{Appendix B. Matlab functions}

\section{B-1 Alate reproduction}

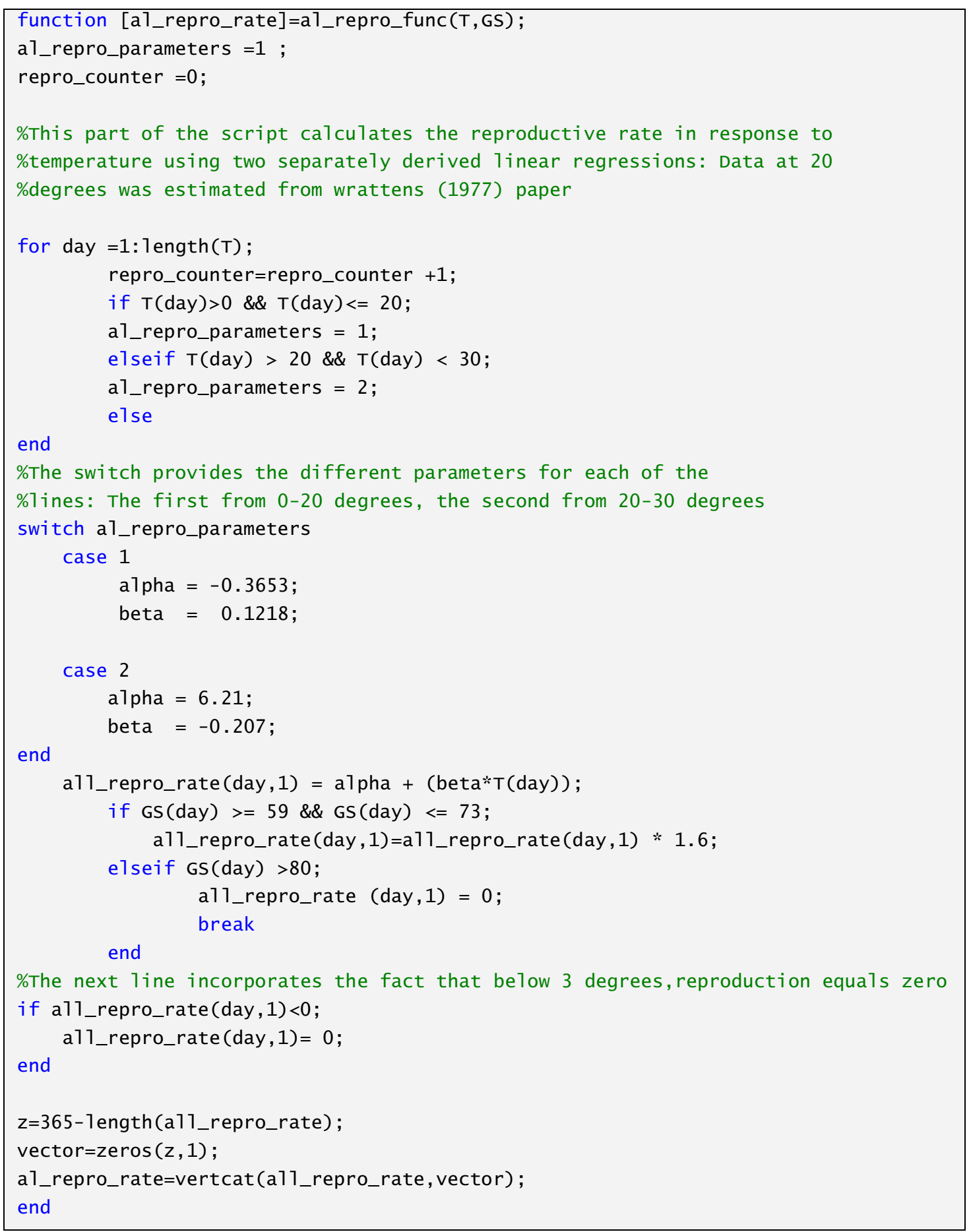




\section{B-2 Apterous reproduction}

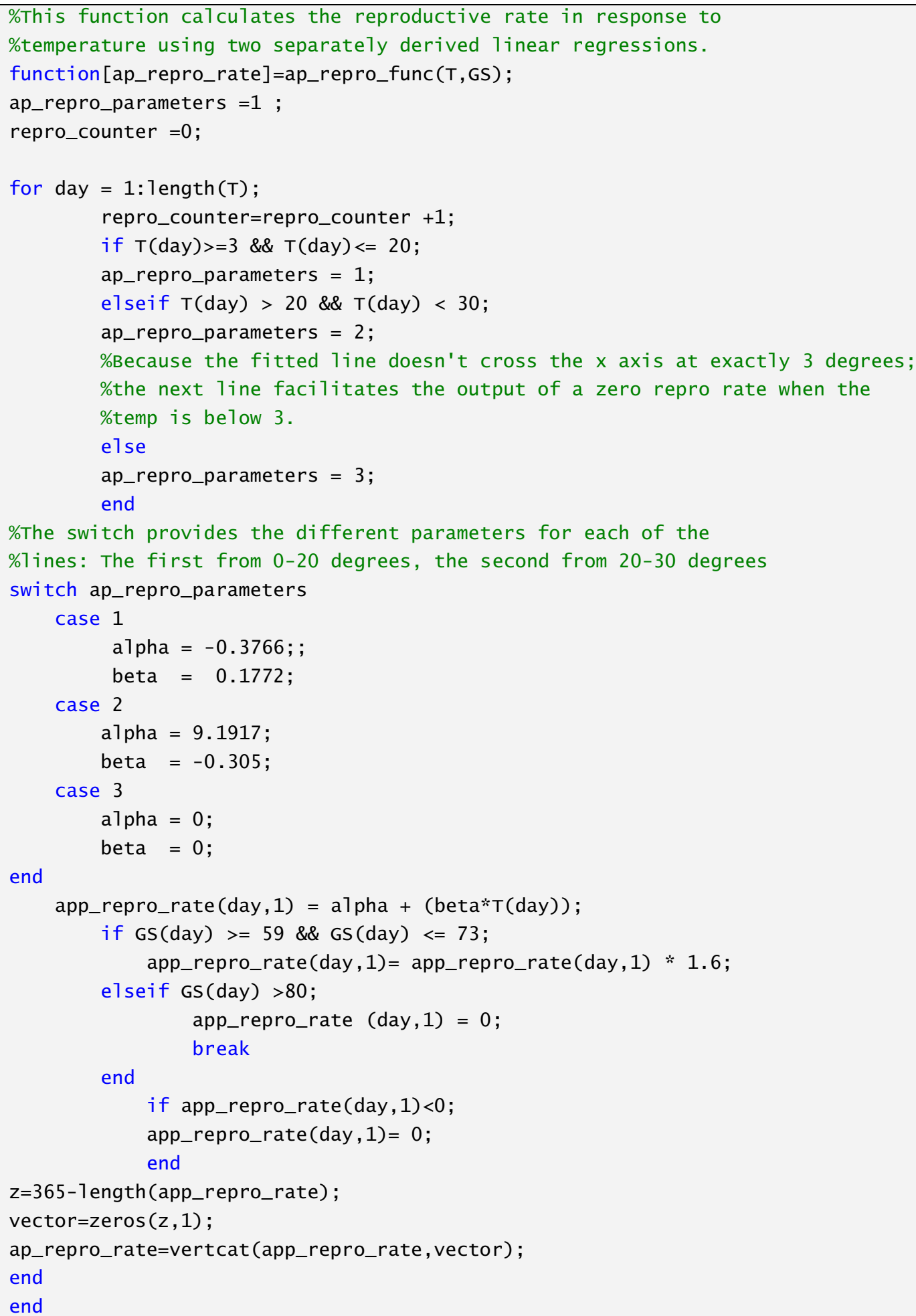




\section{B-3 Alighting}

\%This function applies the concentration and deposition factors to the

\%aphid catches to produce the numbers alighting infield

function [aphids_landing]= alighting(aphids_caught)

\%This line wi11 multiply al1 the aphid numbers by the deposition factor and

\%concentration factor

aphids_1anding $(:, 1)=$ aphids_caught $(:, 1) * 237 * 40$;

end

B-4 Cumulative degree-days

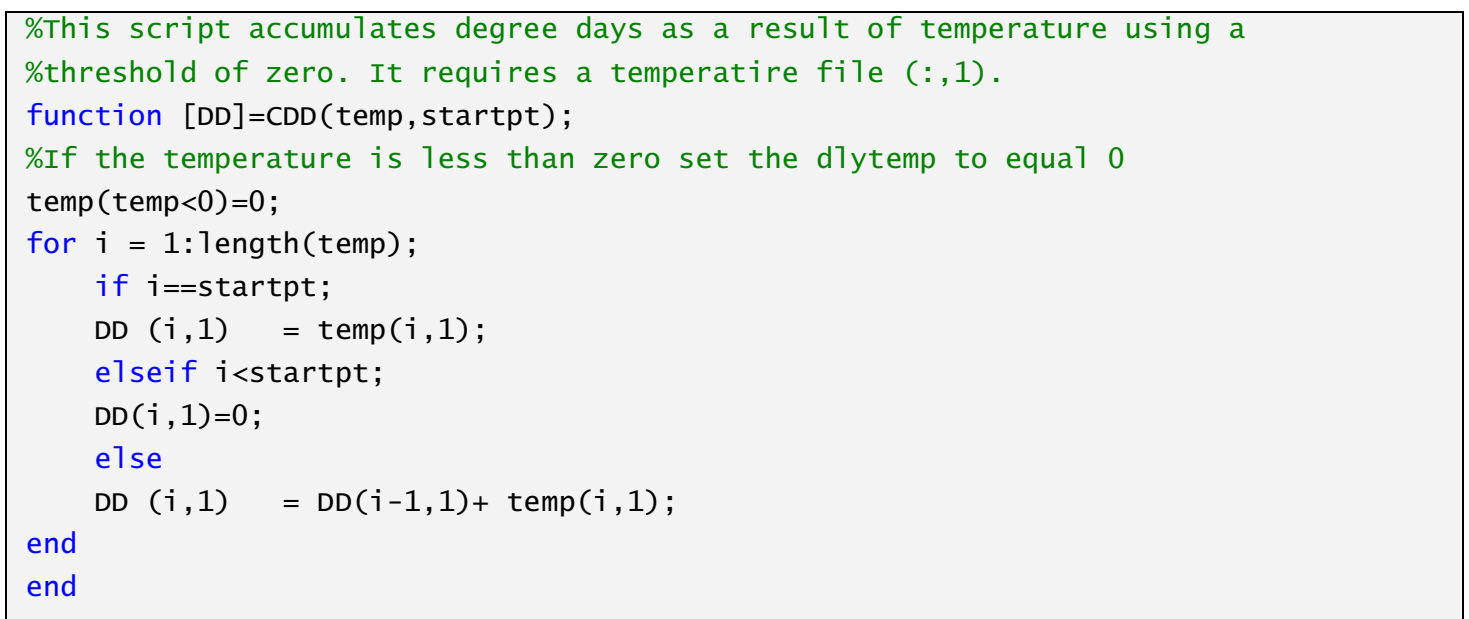

B-5 Growth stage calculation

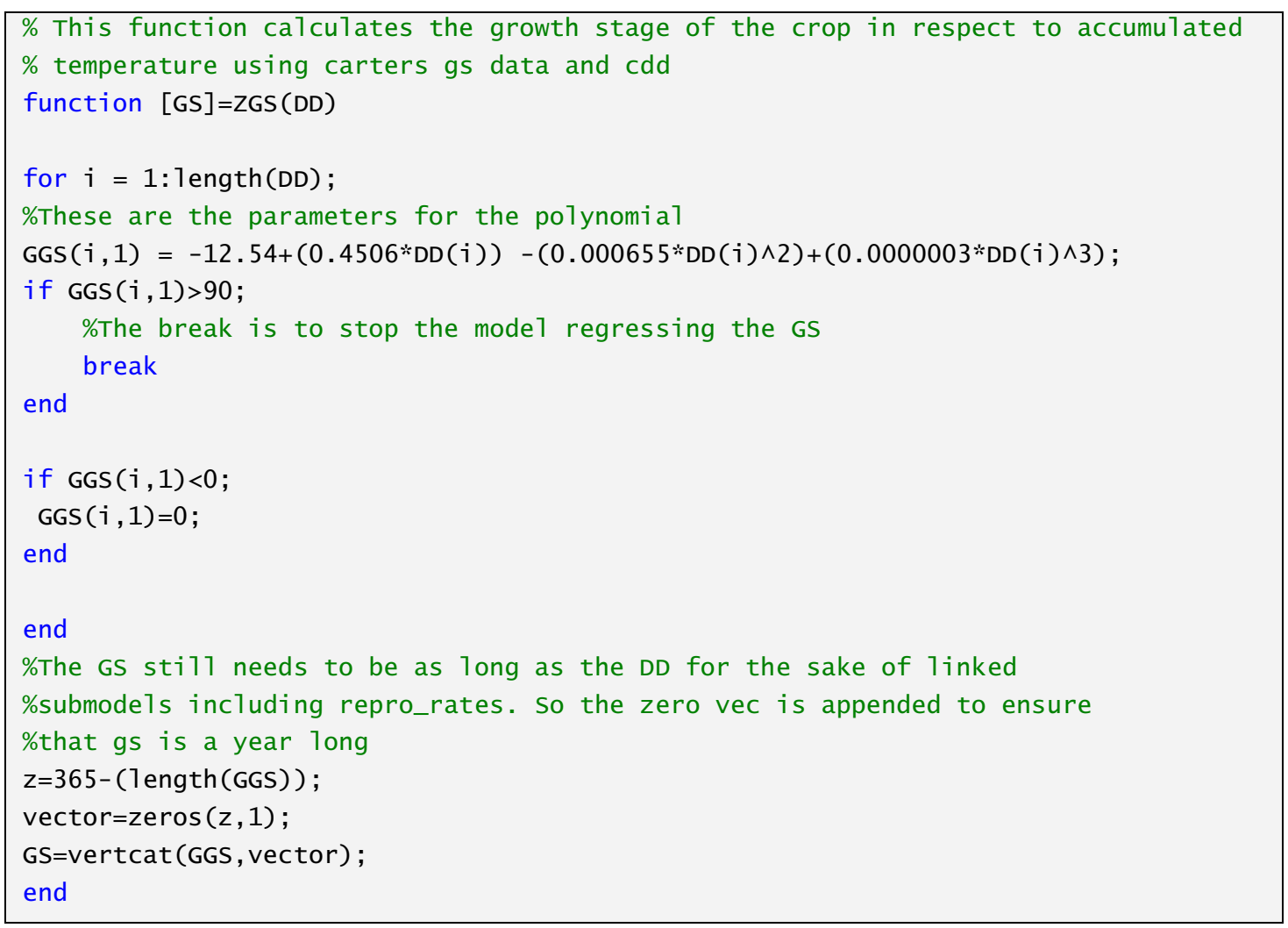




\section{B-6 Calculation of hourly temperatures}

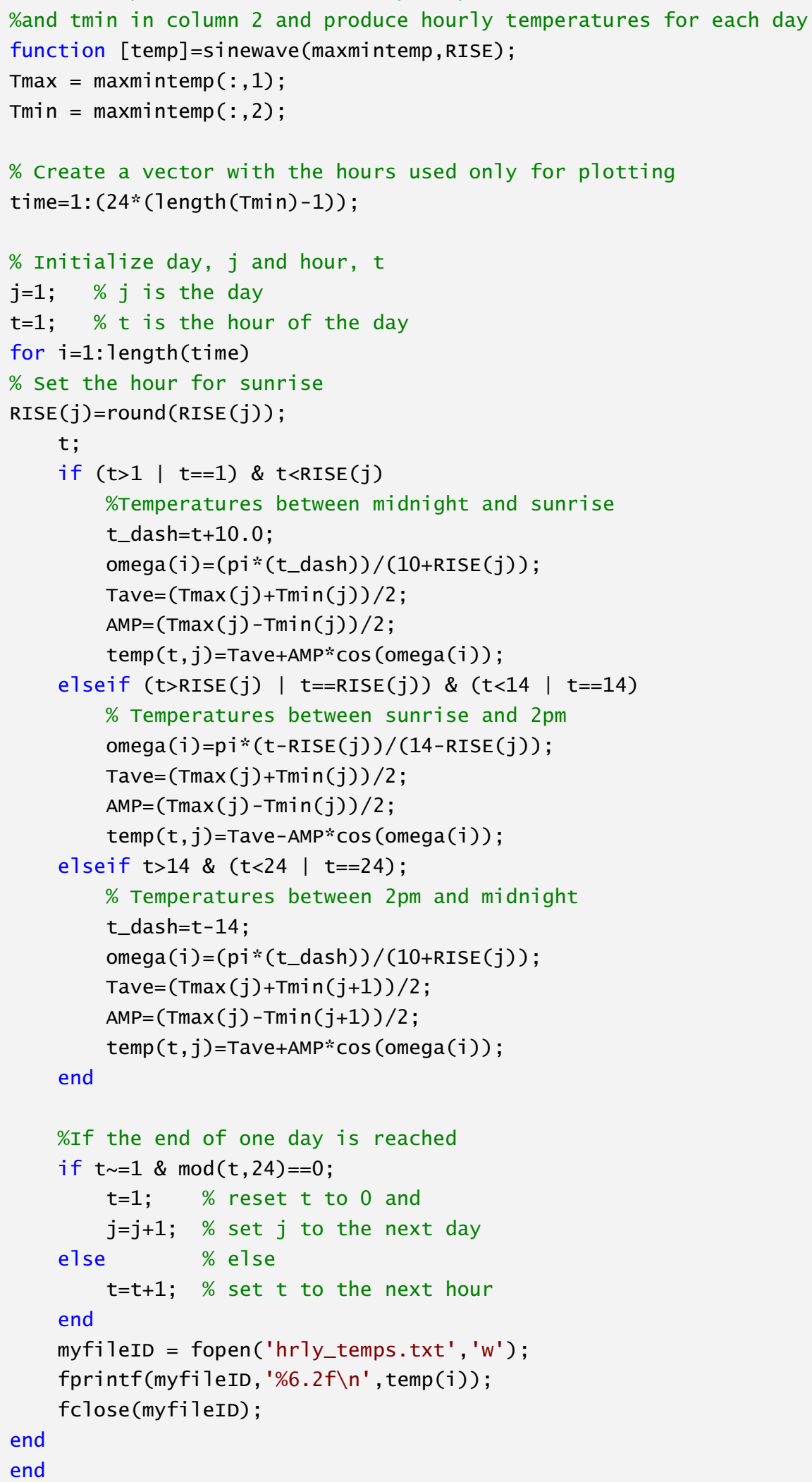




\section{References}

Ankersmit, G.W., Dijkman, H., 1983. Alatae production in the cereal aphid Sitobion avenae. Netherlands J. Plant Pathol. 89, 105-112, http://dx.doi.org/10.1007/ BF01976349.

BADC, 2014. British Atmospheric Data Centre [WWW Document]. Met Off. MIDAS Station. URL http://badc.nerc.ac.uk/search/midas_stations/.

Bale, J.S., 1989. Cold hardiness and overwintering of insects. Agric. Zool. Rev. 3, 157-192.

Begler, C.H., 2008. Suncycle.m [WWW Document]. URL http://mooring.ucsd.edu/ software/matlab/doc/toolbox/geo/suncycle.html.

Cariboni, J., Gatelli, D., Liska, R., Saltelli, A., 2007. The role of sensitivity analysis in ecological modelling. Ecol. Modell. 203, 167-182.

Carter, N., Dixon, A.F.G., Rabbinge, R., 1982. Cereal Aphid Populations: Biology, Simulation and Prediction. Pudoc Wageningen, pp. 97

Carter, N., 1978. A Simulation Study of English Grain Aphid (Sitobion avenae f.) Populations: PhD Thesis. University of East Anglia.

Carter, N., 1985. Simulation modelling of the population dynamics of cereal aphids Biosystems 18, 111-119.

Dean, G.J., 1973. Bionomics of aphids reared on cereals and some Gramineae. Ann. Appl. Biol. 73, 127-135.

Dean, G.J., 1974. Effect of temperature on the cereal aphids Metopolophium dirhodum (Wlk.), Rhopalosiphum padi (L.) and Macrosiphum avenue (F.) (Hem., Aphididae). Bull. Entomol. Res. 63, 401-409.

Dedryver, C.-A., Le Ralec, A., Fabre, F., 2010. The conflicting relationships between aphids and men: a review of aphid damage and control strategies. C. R. Biol. 333, 539-553.

Dewar, A.M., 2014. Alternative insecticides to control grain aphids, Sitobion avenae, that are resistant to pyrethroids. Suffolk.

Entwistle, J.C., Dixon, A.F.G., 1986. Short-term forecasting of peak population density of the grain aphid (Sitobion avenae) on wheat. Ann. Appl. Biol. 109, 215-222.

Foster, S.P., Devine, G., Devonshire, A.L., 2007. Insecticide resistance. In: Van Emden, H.F., Harrington, R. (Eds.), Aphids as Crop Pests. CAB International, Oxfordshire, pp. 261-286.

Gosselke, U., Triltsch, H., Roßberg, D., Freier, B., 2001. GETLAUS01-the latest version of a model for simulating aphid population dynamics in dependence on antagonists in wheat. Ecol. Modell. 145, 143-157.

Hamby, D.M., 1994. A review of techniques for parameter sensitivity analysis of environmental models. Environ. Monit. Assess. 32, 135-154.

Harrington, R., Woiwod, I., 2007. The rothamsted insect survey: foresight from hindsight. Outlooks Pest Manag. 18, 9-14, http://dx.doi.org/10.1564/18feb03.

Hoogenboom, G., Huck, M.G., 1986. Rootsimu v4.0-a Dynamic Simulation of Root Growth, Water Uptake, and Biomass Partitioning in a Soil-plant-atmosphere Continuum: Update and Documentation.

Johnson, J.B., Omland, K.S., 2004. Model selection in ecology and evolution. Trends Ecol. Evol. 19, 101-108.

Katis, I.N., Tsitsipis, J.A., Stevens, M., Powell, G., 2007. Transmission of plant viruses. In: Aphids as Crop Pests., pp. 353

Kendall, D.A., Brain, P., Chinn, N.E., 1992. A simulation model of the epidemiology of barley yellow dwarf virus in winter sown cereals and its application to forecasting. J. Appl. Ecol. 29, 414-426.

Kindlmann, P., Jarošík, V., Dixon, A.F.G., 2007. Population dynamics. In: Van Emden, H.F., Harrington, R. (Eds.), Aphids as Crop Pests. CAB International, Oxfordshire, pp. 311-324.

Kontodimas, D.C., Eliopoulos, P.A., Stathas, G.J., Economou, L.P., 2004. Comparative temperature-dependent development of Nephus includens (Kirsch) and Nephus bisignatus (Boheman) (Coleoptera: coccinellidae) preying on Planococcus citri (Risso) (Homoptera: pseudococcidae): evaluation of a linear and various nonlinear models us. Environ. Entomol. 33, 1-11.

Lactin, D.J., Holliday, N.J., Johnson, D.L., Craigen, R., 1995. Improved rate model of temperature-dependent development by arthropods. Environ. Entomol. 24, 68-75.

Logan, J.A., Wollkind, D.J., Hoyt, S.C., Tanigoshi, L.K., 1976. An analytic model for description of temperature dependent rate phenomena in arthropods. Environ. Entomol. 5, 1133-1140.

Lykouressis, D.P., 1985. Temperature requirements of Sitobion avenae necessary for ecological studies, by assessing methods for the estimation of instar duration. Zeitschrift für Angew. Entomol. 100, 479-493.

Morgan, D., 1996. Towards a Decision Support System for Barley Yellow Dwarf Virus: the Role of Modelling. Central Science Laboratory, Statens Planteavslforsog, pp. 1128-1132.
Morgan, D., 2000. Population dynamics of the bird cherry-oat aphid, Rhopalosiphum padi (L.), during the autumn and winter: a modelling approach. Agric. For. Entomol. 2, 297-304, http://dx.doi.org/10.1046/j. 14619563.2000.00079.x.

Newton, C., Dixon, A.F.G., 1990a. Pattern of growth in weight of alate and apterous nymphs of the English grain aphid, Sitobion avenae. Entomol. Exp. Appl. 55, 231-238.

Newton, C., Dixon, A.F.G., 1990b. Embryonic growth rate and birth weight of the offspring of apterous and alate aphids: a cost of dispersal. Entomol. Exp. Appl. 55, 223-230.

Plantegenest, M., Pierre, J.S., Dedryver, C.A., Kindlmann, P., 2001. Assessment of the relative impact of different natural enemies on population dynamics of the grain aphid Sitobion avenae in the field. Ecol. Entomol. 26, 404-410, http://dx. doi.org/10.1046/j.1365-2311.2001.00330.x.

Poehling, H.M., Freier, B., Klüken, A.M., 2007. IPM case studies: grain. In: Van Emden, H.F., Harrington, R. (Eds.), Aphids as Crop Pests. CAB International, Oxfordshire, pp. 597-606.

Rabbinge, R., Ankersmit, G.W., Pak, G.A., 1979. Epidemiology and simulation of population development of Sitobion avenae in winter wheat. Neth. J. Plant Pathol. 85, 197-220.

Rautapaa, J., 1966. The effect of the English grain aphid Macrosiphum avenae (F.) (Homoptera, Aphididae), on the yield and quality of wheat. Ann. Agric. Fenn. 5 , 334-341.

Reicosky, D.C., Winkelman, L.J., Baker, J.M., Baker, D.G., 1989. Accuracy of hourly air temperatures calculated from daily minima and maxima. Agric. For. Meteorol. 46, 193-209.

Skirvin, D.J., Perry, J.N., Harrington, R., 1997. A model describing the population dynamics of Sitobion avenae and Coccinella septempunctata. Ecol. Modell. 96, 29-39, http://dx.doi.org/10.1016/s0304-3800(96)00048-8.

Skirvin, D.J., 1995. Simulating the effects of climate change on Sitobion avenae F. (Homoptera: Aphididae) and Cocinella septempunctata L. (Coleoptera: PhD Thesis. University of Notingham.

Stinner, R.E., Gutierrez, A.P., Butler Jr, G.D., 1974. An algorithm for temperature-dependent growth rate simulation. Can. Entomol. 106, 519-524, http://dx.doi.org/10.4039/Ent106519-5.

Sutherland, O.R.W., 1969. The role of the host plant in the production of winged form by two strains of the pea aphid, Acyrthosiphon pisum. J. Insect Physiol. 15, 2179-2201.

Taylor, L.R., Palmer, J.M.P., 1972. Aerial sampling. In: Van Emden, H.F. (Ed.), Aphid Technology. Academic Press, London and New York, pp. 189-229.

Teulon, D.A.J., Stufkens, M.A.W., Fletcher, J.D., 2004. Crop infestation by aphids is related to flight activity detected with 7.5 metre high suction traps. New Zeal. Plant Prot. 57, 227-232.

Thackray, D.J., Diggle, A.J., Jones, R.A.C., 2009. BYDV PREDICTOR: a simulation model to predict aphid arrival, epidemics of Barley yellow dwarf virus and yield losses in wheat crops in a Mediterranean-type environment. Plant Pathol. 58, 186-202, http://dx.doi.org/10.1111/j.1365-3059.2008.01950.x.

Union, E., parliament and council of the european, 2012. Plant protection products. Ireland.

Wagner, T.L., Wu, H.-I., Sharpe, P., Schoolfield, R.M., Coulson, R.N., 1984. Modeling insect development rates: a literature review and application of a biophysical model. Ann. Entomol. Soc. Am. 77, 208-220.

Walgama, R.S., Zalucki, M.P., 2006. Evaluation of different models to describe egg and pupal development of Xyleborus fornicatus Eichh. (Coleoptera Scolytidae), the shot hole borer of tea in Sri Lanka. Insect Sci. 13, 109-118.

Watt, A.D., Dixon, A.F.G., 1981. The role of cereal growth stages and crowding in the induction of alatae in Sitobion avenae and its consequences for population growth. Ecol. Entomol. 6, 441-447.

Watt, A.D., 1979. The effect of cereal growth stages on the reproductive activity of Sitobion avenae and Metopolophium dirhodum. Ann. Appl. Biol. 91, 147-157.

Williams, C.T., Wratten, S.D., 1987. The winter development, reproduction and lifespan of viviparae of Sitobion avenae (F.) (Hemiptera: Aphididae) on wheat in England. Bull. Entomol. Res. 77, 19-34.

Wratten, S.D., 1977. Reproductive strategy of winged and wingless morphs of the aphids Sitobion avenae and Metopolophium dirhodum. Ann. Appl. Biol. 85, 319-331.

Zadoks, J.C., Chang, T.T., Konzak, C.F., 1974. A decimal code for the growth stages of cereals. Weed Res. 14, 415-421, http://dx.doi.org/10.1111/j.1365-3180.1974. tb01084.x.

Zhou, X., Carter, N., Mumford, J., 1989. A simulation model describing the population dynamics and damage potential of the rose grain aphid, Metopolophium dirhodum (Walker) (Hemiptera: Aphididae), in the UK. Bull. Entomol. Res. 79, 373-380. 